\title{
Semi-inclusive wino and higgsino annihilation to $\mathrm{LL}^{\prime}$
}

\author{
Matthew Baumgart ${ }^{a, b}$ and Varun Vaidya ${ }^{a}$ \\ ${ }^{a}$ Department of Physics, Carnegie Mellon University, \\ Pittsburgh, PA 15213, U.S.A. \\ ${ }^{b}$ New High Energy Theory Center, Rutgers University, \\ Piscataway, NJ 08854, U.S.A. \\ E-mail: baumgart@physics.rutgers.edu, vpvaidya@andrew.cmu.edu
}

ABSTRACT: We systematically compute the annihilation rate for winos and higgsinos into the final state relevant for indirect detection experiments, $\gamma+X$. The radiative corrections to this process receive enhancement from the large Bloch-Nordsieck-Violating Sudakov logarithm, $\log \left(2 M_{\chi} / M_{W}\right)$. We resum the double logs and include single logs to fixed order using a formalism that combines nonrelativistic and soft-collinear effective field theories. For the wino case, we update an earlier exclusion adapting results of the HESS experiment. At the thermal relic mass of $3 \mathrm{TeV}, \mathrm{LL}^{\prime}$ corrections result in a $\sim 30 \%$ reduction in rate relative to LL. Nonetheless, single logs do not save the wino, and it is still excluded by an order of magnitude. Experimental cuts produce an endpoint region which, our results show, significantly effects the higgsino rate at its thermal-relic mass near $1 \mathrm{TeV}$ and is deserving of further study.

Keywords: NLO Computations, Supersymmetry Phenomenology

ArXiv EPrint: 1510.02470 


\section{Contents}

1 Introduction 1

2 Effective field theory of heavy WIMP annihilation 3

2.1 Wino operators 4

2.2 Wino anomalous dimensions 5

2.3 Resummed wino annihilation rate 8

$\begin{array}{llr}3 & \text { Higgsino } & 10\end{array}$

$\begin{array}{lll}3.1 & \text { Anomalous dimensions } & 12\end{array}$

$\begin{array}{lll}3.2 & \text { Resummed higgsino annihilation rate } & 14\end{array}$

4 Dark matter constraints and conclusion $\quad 17$

$\begin{array}{ll}\text { A Neutralino masses and mixing } & 20\end{array}$

B Sommerfeld enhancement $\quad 22$

B.1 Wino 22

B.2 Higgsino 22

$\begin{array}{ll}\text { C Photon wavefunction renormalization } & 23\end{array}$

\section{Introduction}

While the Standard Model (SM) lacks a viable dark matter (DM) candidate, it is straightforward to extend it to a theory that possesses one. The fact that a weak-scale particle charged under the electroweak group can simply freeze out of the primordial plasma with the correct relic abundance has been dubbed the "WIMP Miracle" (cf. [1]). Since the dynamics that set the electroweak scale are presently unknown, it is natural to go a step further and propose that WIMP (Weakly-Interacting Massive Particle) dark matter arises from the sector that solves the hierarchy problem. Supersymmetry (SUSY) is the classic example of this 'two-birds with one stone' approach to beyond the Standard Model physics. However, the absence of superpartner discovery at the LHC and elsewhere has brought the public perception of the theory into some disrepute. One may counter though, that this very notion of SUSY's having a successful, simple DM candidate predicts precisely the current experimental situation. The dark matter is necessarily the lightest supersymmetric particle (LSP), and its most straightforward realization is the neutralino. In the absence of large mixing, we can discuss each of the three neutralino states as its own case. The bino overcloses the universe in the absence of nearly-degenerate sfermions. This leaves wino and higgsino, the two cases we consider here. If we want them to be the advertised thermal 
relic, this fixes their masses to be $M_{\text {wino }} \equiv\left(M_{\chi}\right)=2.7-2.9 \mathrm{TeV}$ or $M_{\text {higgsino }} \equiv\left(M_{\chi}\right)=$ $1 \mathrm{TeV}[2-4]$. Thus, the simple SUSY dark matter story has an electroweak LSP at or above $1 \mathrm{TeV}$, and thus has no observable collider signature for the foreseeable future.

While $\mathrm{TeV}$-scale, weakly-interacting states are out of range of the current generation of colliders, they represent a big target of opportunity for indirect detection experiments. Observation of a nearly monochromatic line of photons at these energies would be a smoking gun of new physics. The possibility of such experiments to set stringent limits on the wino scenario was explored in $[2,5]$. By combining a numerical calculation of the Sommerfeld enhancement with a tree-level annihilation of WIMPs to $\gamma \gamma+\frac{1}{2} \gamma Z$, they found that the HESS experiment ruled out the wino by a factor of 15 under the assumption of an NFW dark matter halo profile [6]. In particular though, [5] extended their annihilation calculation to one-loop and found a reduction in rate by $\sim 4 \times$ at the thermal relic mass of $3 \mathrm{TeV}$. In order to test such a large radiative correction, in our previous papers $([7,8])$, we developed an effective field theory (EFT) approach to resum the large Sudakov double-logs that appear, $\log \left(2 M_{\chi} / M_{W}\right)$, factorizing their contribution from the Sommerfeld factors. Although the gauge bosons in the theory are electroweak, the approach is based on two different EFTs developed for QCD, non-relativistic QCD (NRQCD) [9] and soft-collinear effective theory (SCET) [10-13]. Since the experiment only detects one of the hard photons from annihilation and its resolution is on the order of a few hundred $\mathrm{GeV}$, we computed the semi-inclusive annihilation rate, $\chi^{0} \chi^{0} \rightarrow \gamma+X$. We found the overall correction from resummed double logs to be modest, with a few percent reduction in the cross section at the relic density mass of $3 \mathrm{TeV}$ relative to tree level (+ Sommerfeld enhancement). Thus, the factor of $\sim 15$ exclusion by HESS remained, unless one invoked a profile with coring $\gtrsim 1.5 \mathrm{kpc}$, in tension with recent simulations [14-16].

In between the release of our two papers, two other groups produced calculations using effective field theory for wino annihilation $[17,18]$ to $\gamma \gamma+\frac{1}{2} \gamma Z .{ }^{1}$ Both papers reported a much larger reduction, getting roughly half the rate found at tree level. This need not be a contradiction as our semi-inclusive cross section necessarily includes more processes. Nonetheless, to improve our earlier, leading log (LL) resummed result, we now compute the single-log contributions. Despite being formally subleading in the power counting parameter, working at single-log order brings in new effects which have the potential to be large: 1) the possibility of the photon to fragment into pairs of SM particles, 2) accounting for real-emission processes that make $E_{\gamma}$ so much less than $M_{\chi}$ that events wind up outside the signal bin. We will find, in fact, that 2) is numerically important. While this is not enough to prevent the wino from being ruled out by HESS by an order of magnitude, it does prompt us to undertake a future study to calculate endpoint logarithms which are different from the $\log \left(2 M_{\chi} / M_{W}\right)$ contributions we have been including and resumming thus far [20]. We also extend our formalism to calculate the semi-inclusive rate of higgsino annihilation. Since the thermal relic mass is just $1 \mathrm{TeV}$, the log expansion is less accurate and we will see that single logs introduce a sizable correction.

\footnotetext{
${ }^{1}$ Ref. [17] actually treated an SU(2) scalar triplet, but as we are in the nonrelativistic limit and only annihilating a single spin channel in the fermion case, the calculation is identical.
} 


\begin{tabular}{|c|c|c|}
\hline \multirow{2}{*}{$\left(p^{+}, p^{-}, p_{\perp}\right)$} & $B_{n}^{\perp}:$ Collinear & $S_{n} \supset$ Soft \\
\cline { 2 - 3 } & $M_{\chi}\left(1, \lambda^{2}, \lambda\right)$ & $M_{\chi}(\lambda, \lambda, \lambda)$ \\
\hline $\begin{array}{c}\text { NRQCD } \\
(\mathrm{E}, \mathrm{p})\end{array}$ & Potential & $\chi:$ WIMP \\
\cline { 2 - 3 } & $\left(M_{\chi} v^{2}, M_{\chi} v\right)$ & $\left(M_{\chi} v^{2}, M_{\chi} v\right)$ \\
\hline
\end{tabular}

Table 1. Momentum scalings for the EFT fields. The SCET field momenta are in light conecoordinates. The collinear gauge boson and WIMP fields appear explicitly in our operators, so we have included our notation for them below. Soft gauge bosons, $n \cdot A_{n}$, enter our calculation through soft Wilson lines. Since we solve for the Sommerfeld enhancement with an instantaneous potential, this accounts for the action of the potential modes.

In section 2, we briefly review the EFT for WIMP annihilation to $\gamma+X$, and present our previous results for the wino with LL resummation along with single-log contributions at fixed order, providing an LL' result. Section 3 extends this to the higgsino. In section 4, we give the plots of the cross section as a function of the WIMP mass. While the splitting between charged and neutral wino states are fairly model independently around $170 \mathrm{MeV}$, we give plots for the higgsino-case in two different limits of mass splittings. We also discuss the importance of corrections arising out of finite detector resolution and ways that these can be handled in a controlled manner. In the appendix, we collect some technical results on the neutralino mass matrix in the MSSM, Sommerfeld enhancement, and our treatment of the photon fragmentation down to zero invariant mass.

\section{Effective field theory of heavy WIMP annihilation}

We lay out the details of our effective field theory approach and factorization theorem in $[7,8]$. The EFT is a hybrid of NRQCD and SCET. ${ }^{2}$ The former is necessary to handle the heavy, slowly-moving initial state and is organized as a power counting in $v$, the WIMP velocity. The latter resums the large kinematic logarithms that arise from our highlyboosted final state particles, with a power-counting in $\lambda=M_{W} / M_{\chi}$. One immediate benefit of this approach is that it disentangles the different physical effects arising from the same fields in the underlying theory. Since we are quantifying the nonperturbative physics of both Sommerfeld enhancement and Sudakov resummation and since loops of $W$ boson contribute to both, the EFT makes it manifest which nontrivial process a given diagram is contributing to. In table 1, we list the effective theory fields and their power counting.

Additionally, we note that for the experiments under consideration, resolution of the photon signal is sufficiently poor that we cannot distinguish between strict two-body $(\gamma \gamma$ or $\gamma \mathrm{Z}$ ) annihilation and processes where the photon either recoils against or emits soft or collinear $W \mathrm{~s}$. For this reason, we make use of the Operator Product Expansion (OPE) to calculate the semi-inclusive annihilation rate $\sigma\left(\chi^{0} \chi^{0}\right) \rightarrow \gamma+X$ (cf. figure 1). The recoil state will not lead to any IR divergences dependent on $M_{W}$, even at the single log level. Since we are totally inclusive in the recoil- (or $\bar{n}$-) sector, and since the initial state has no $\bar{n}$

\footnotetext{
${ }^{2}$ The SCET we use is SCET-II, as soft and collinear fields have the same virtuality. This is because $M_{W}$, which sets the collinear scale, also provides an IR cutoff, beyond which soft $W$ s cannot fall.
} 


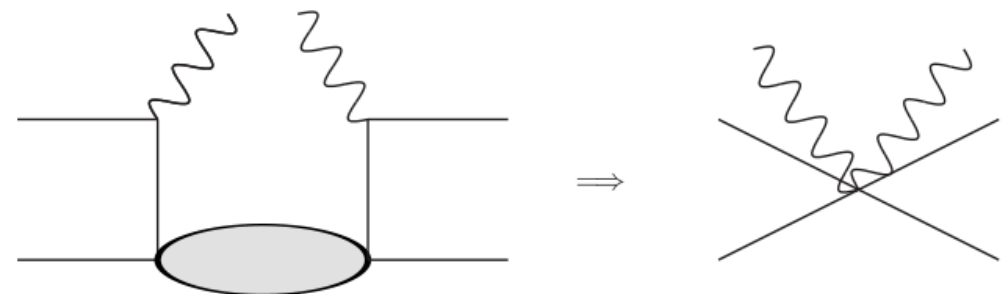

Figure 1. Integrating out the recoil jet. The open curly lines correspond to the observed photon which is accompanied by any amount of soft or collinear radiation.

particles, by taking the OPE, we are calculating a recoil-sector vacuum expectation value. Thus, the only operator in the expansion that can contribute to the vev is proportional to the identity and is therefore IR safe. ${ }^{3}$ This can also be seen by considering the the recoil jet content, which has $\operatorname{soft}\left(p_{s}\right)$ as well as $\bar{n}$ collinear $\left(p_{\bar{n}}\right)$ radiation. The invariant mass of this recoil would then have momentum scaling $p_{s}+p_{\bar{n}} \sim M_{\chi}(\lambda, 1, \lambda)$ which has an invariant mass of $M_{\chi}^{2} \lambda$. On the other hand, our EFT is designed to integrate out any modes (by doing an OPE) with a virtuality greater than $\lambda^{2} M_{\chi}^{2}$. We can perform the OPE by matching to the semi-inclusive rate to produce a photon in the full theory at the scale $2 M_{\chi}$. The procedure is identical in both the wino and higgsino cases, but the different representations of these states, along with the higgsino's nonzero hypercharge, give us a different basis of operators. We now discuss both cases.

\subsection{Wino operators}

The first case we consider is that of a wino-like neutralino. This covers the possibility of dark matter arising as the LSP in the MSSM (or an extension) as long as it is sufficiently split from other states that we can ignore the effects of coannihilation and mixing. Operationally, though, we are simply adding an $\mathrm{SU}(2)$ triplet to the $\mathrm{SM}$, and so this scenario could arise as a more minimal model of DM. We will nonetheless refer to this state as the wino. The details of the operator basis and factorization theorem along with the analysis of the cross-section at leading log were presented in [7,8]. Here we briefly review this case and extend the analysis to $L L^{\prime}$, including the single-log contributions as fixed order corrections to the resummed double log. The operator basis dressed with Wilson lines is

$$
\begin{aligned}
O_{1}= & \left(\bar{\chi} \gamma^{5} \chi\right)|0\rangle\langle 0|\left(\bar{\chi} \gamma^{5} \chi\right) B^{A} B^{A} \\
O_{2}= & \frac{1}{2}\left\{\left(\bar{\chi} \gamma^{5} \chi\right)|0\rangle\left\langle 0\left|\left(\bar{\chi}_{A^{\prime}} \gamma^{5} \chi_{B^{\prime}}\right)+\left(\bar{\chi}_{A^{\prime}} \gamma^{5} \chi_{B^{\prime}}\right)\right| 0\right\rangle\langle 0|\left(\bar{\chi} \gamma^{5} \chi\right)\right\} B^{\tilde{A}} B^{\tilde{B}} \\
& S_{v A^{\prime} A}^{\top} S_{v B B^{\prime}} S_{n \tilde{A} A}^{\top} S_{n B \tilde{B}} \\
O_{3}= & \left(\bar{\chi}_{C} \gamma^{5} \chi_{D}\right)|0\rangle\langle 0|\left(\bar{\chi}_{D} \gamma^{5} \chi_{C}\right) B^{A} B^{A} \\
O_{4}= & \left(\bar{\chi}_{A^{\prime}} \gamma^{5} \chi_{C}\right)|0\rangle\langle 0|\left(\bar{\chi}_{C} \gamma^{5} \chi_{B^{\prime}}\right) B^{\tilde{A}} B^{\tilde{B}} S_{v A^{\prime} A}^{\top} S_{v B B^{\prime}} S_{n \tilde{A} A}^{\top} S_{n B \tilde{B}} .
\end{aligned}
$$

The vacuum insertion approximation enforces that the WIMP fields annihilate the initial state and holds up to corrections of $\mathcal{O}\left(v^{2}\right)$. In general, we will drop the vacuum projector.

\footnotetext{
${ }^{3}$ We thank Ira Rothstein for this argument [19].
} 
There is also an implicit Lorentz contraction and projection of the final state onto a singlephoton state in the $n$ direction,

$$
B_{n \mu}^{\perp A} B_{n}^{\perp \mu B} \equiv \sum_{X} B_{n \mu}^{\perp A}|\gamma+X\rangle\langle\gamma+X| B_{n}^{\perp \mu B},
$$

and without ambiguity, we drop the $n$ and $\perp$ from $B_{n}^{\perp A}$. In preparation for computing the anomalous dimensions, it is useful to decompose the operators in eq. (2.1) into the soft and collinear sectors,

$$
\begin{aligned}
O_{s}^{a} & =S_{v A^{\prime} A^{T}}^{T} S_{v B B^{\prime}} S_{n \tilde{A} A}^{T} S_{n B \tilde{B}}, \quad O_{s}^{b}=\delta_{\tilde{A} \tilde{B}} \delta_{A^{\prime} B^{\prime}} \\
O_{c}^{a} & =B_{\tilde{A}} B_{\tilde{B}} \\
O_{c}^{b} & =B_{D} B_{D} \delta_{\tilde{A} \tilde{B}} .
\end{aligned}
$$

The cross-section in terms of this basis is

$$
\begin{aligned}
\frac{1}{E_{\gamma}} \frac{d \sigma}{d E_{\gamma}}= & \frac{1}{4 M_{\chi}^{2} v}\left\langle 0\left|O_{s}^{a}\right| 0\right\rangle\left[\int d n \cdot p \left\{C _ { 2 } ( M _ { \chi } , n \cdot p ) \langle p _ { 1 } p _ { 2 } | \frac { 1 } { 2 } \left\{\left(\bar{\chi} \gamma^{5} \chi\right)\left(\bar{\chi}_{A^{\prime}} \gamma^{5} \chi_{B^{\prime}}\right)\right.\right.\right. \\
& \left.+\left(\bar{\chi}_{A^{\prime}} \gamma^{5} \chi_{B^{\prime}}\right)\left(\bar{\chi} \gamma^{5} \chi\right)\right\}(0)\left|p_{1} p_{2}\right\rangle \\
& \left.\left.+C_{4}\left(M_{\chi}, n \cdot p\right)\left\langle p_{1} p_{2}\left|\left(\bar{\chi}_{A^{\prime}} \gamma^{5} \chi_{C}\right)\left(\bar{\chi}_{C} \gamma^{5} \chi_{B^{\prime}}\right)(0)\right| p_{1} p_{2}\right\rangle\right\} F_{\tilde{A} \tilde{B}}^{\gamma}\left(\frac{2 E_{\gamma}}{n \cdot p}\right)\right] \\
& +\left[\int d n \cdot p \left\{C_{1}\left(M_{\chi}, n \cdot p\right)\left\langle p_{1} p_{2}\left|\left(\bar{\chi} \gamma^{5} \chi\right)\left(\bar{\chi} \gamma^{5} \chi\right)(0)\right| p_{1} p_{2}\right\rangle+C_{3}\left(M_{\chi}, n \cdot p\right)\right.\right. \\
& \left.\left.\times\left\langle p_{1} p_{2}\left|\left(\bar{\chi}_{C} \gamma^{5} \chi_{D}\right)\left(\bar{\chi}_{D} \gamma^{5} \chi_{C}\right)(0)\right| p_{1} p_{2}\right\rangle\right\} F_{\gamma}\left(\frac{2 E_{\gamma}}{n \cdot p}\right)\right],
\end{aligned}
$$

where $F_{\tilde{A} \tilde{B}}^{\gamma}$ is a fragmentation function defined by

$$
\begin{aligned}
F_{\tilde{A} \tilde{B}}^{\gamma}\left(\frac{n \cdot k}{n \cdot p}\right)= & \int \frac{d x_{-}}{2 \pi} e^{i n \cdot p x_{-}}\left\langle 0\left|B_{\tilde{A}}^{\perp \mu}\left(x_{-}\right)\right| \gamma\left(k_{n}\right)+X_{n}\right\rangle \\
& \times\left\langle\gamma\left(k_{n}\right)+X_{n}\left|B_{\mu \tilde{B}}^{\perp}(0)\right| 0\right\rangle,
\end{aligned}
$$

where $n \cdot p$ is the total +-momentum flowing in the $n$-direction and $F_{\gamma}=F_{\tilde{A} \tilde{B}}^{\gamma} \delta_{\tilde{A} \tilde{B}}$. The $C_{i}$ are just the Wilson coefficients of the full operators given in eq. (2.1). Part of the their functional dependence constrains $n \cdot p=2 M_{\chi}$, from the expansion of the overall momentum-conserving $\delta$-function for WIMP annihilation. As we will detail below, there is a simple relation between the Wilson coefficients at tree level that allows us to determine them at that order by a single calculation.

\subsection{Wino anomalous dimensions}

The advantage of working with the basis in eq. (2.3) becomes apparent when we work at loop level. We can consider the soft and collinear sectors separately. However, soft and collinear modes have the same virtuality and hence the divergences that arise from 

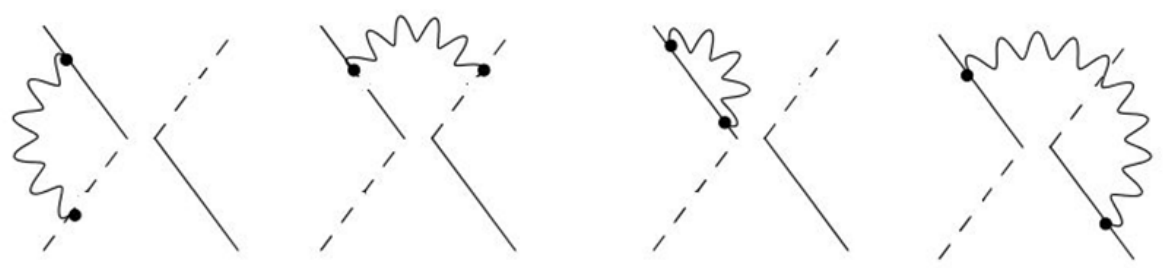

Figure 2. The diagrams contributing to $O_{s}^{a}$. The solid/dashed lines indicate time/light-like Wilson lines.

the factorization of the soft sector from the collinear cannot be regulated by dimensional regularization, which respects boost symmetry. Hence, we need to introduce a rapidity regulator, which manifestly breaks boosts [21,22]. This requires a corresponding factorization scale which we call $\nu$. As it arises from our artificial distinction between soft and collinear modes, it will necessarily drop out of the anomalous dimensions of the full operators in eq. (2.1), after we recombine the separate soft and collinear operators of eq. (2.3) to make them. This provides a useful cross check on the calculation.

We now calculate the matrix elements of the operators defined above, including the single log contributions. The diagrams contributing to the operator $O_{s}^{a}$ are shown in figure 2 .

$$
\begin{aligned}
\left\langle 0\left|O_{s}^{a}\right| 0\right\rangle= & \delta_{A^{\prime} \tilde{A}} \delta_{B^{\prime} \tilde{B}}+\left\{\delta_{\tilde{A} \tilde{B}} \delta_{A^{\prime} B^{\prime}}-3 \delta_{A^{\prime} \tilde{A}} \delta_{B^{\prime} \tilde{B}}\right\} \\
& \times \frac{\alpha_{W}}{\pi}\left[2 \log \left(\frac{\nu}{\mu}\right) \log \left(\frac{\mu}{M_{W}}\right)+\log ^{2}\left(\frac{\mu}{M_{W}}\right)-\log \left(\frac{\mu}{M_{W}}\right)\right]
\end{aligned}
$$

Thus, we radiatively generate the singlet color structure which demonstrates mixing between the singlet and nonsinglet operators in the soft sector.

The diagrams for the operator $O_{c}^{a}$ are shown in figure 3

$$
\begin{aligned}
\left\langle 0\left|O_{c}^{a}\right| 0\right\rangle= & 2 \sin ^{2} \theta_{W}\left\{\delta_{\tilde{A} 3} \delta_{\tilde{B} 3}+\left\{\delta_{\tilde{A} \tilde{B}}-3 \delta_{\tilde{A} 3} \delta_{\tilde{B} 3}\right\} \frac{\alpha_{W}}{\pi}\left\{2 \log \left(\frac{M_{\chi}}{\nu}\right) \log \left(\frac{\mu}{M_{W}}\right)\right\}\right. \\
& \left.+\delta_{\tilde{A} 3} \delta_{\tilde{B} 3} \frac{\alpha_{W}}{2 \pi} \beta_{0} \log \left(\frac{\mu}{M_{W}}\right)+\left(\delta_{\tilde{A} \tilde{B}}-\delta_{\tilde{A} 3} \delta_{\tilde{B} 3}\right) \frac{\alpha_{W}}{\pi} \int_{z_{\text {cut }}}^{1} d z P_{g g}^{*}(z) \log \left(\frac{\mu}{M_{W}}\right)\right\}
\end{aligned}
$$

where,

$$
P_{g g}^{*}(z)=2\left[z(1-z)+\frac{z}{(1-z)^{+}}+\frac{1-z}{z}\right]
$$

with $z$ the energy fraction of $M_{\chi}$ carried by the photon and $z_{\text {cut }}$ is the threshold value for the photon to be detected by the experiment. For HESS, $z_{\text {cut }}$ varies between 0.89 to 0.83 over the range of masses $0.5-19 \mathrm{TeV}$., so we adopt an average value of $z_{\text {cut }}=0.85$. The dependence on $z_{\text {cut }}$ appears because of the semi-inclusive nature of our calculation. We are implicitly including three-body processes where the observed photon has lost a finite amount of its energy. In a forthcoming work [20], we will calculate the dependence of the rate on the possibly numerically important quantity $\log \left(1-z_{\text {cut }}\right)$. Finally, $\beta_{0}=\frac{19}{6}$ is the one-loop $\mathrm{SU}(2) \beta$-function coefficient. 

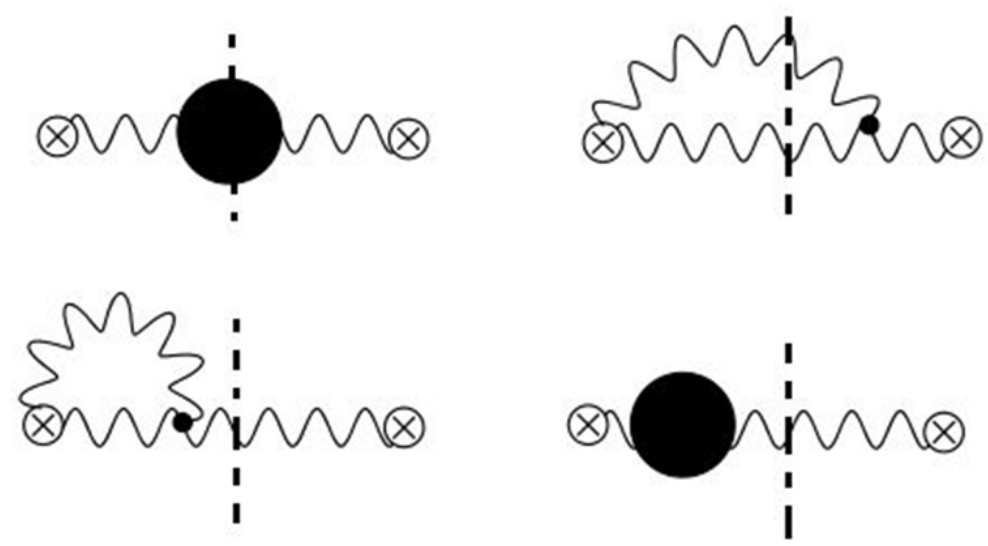

Figure 3. The diagrams contributing to $O_{c}^{a}$. The dark blob contains fermion, scalar and gauge boson loops.

The operator $O_{s}^{b}$ has a trivial structure and hence does not receive radiative corrections, meaning its anomalous dimension is 0 . At one loop, the diagrams that contribute to the $O_{c}^{b}$ matrix element are the same as those for $O_{c}^{a}$ in figure 3. However, $O_{c}^{b}$ is a color singlet, meaning the real and virtual double poles cancel, so it does not have any double logs. Nonetheless, it does have a non-cusp, one-loop anomalous dimension.

$$
\begin{aligned}
& \left\langle 0\left|O_{s}^{b}\right| 0\right\rangle=\delta_{\tilde{A} \tilde{B}} \delta_{A^{\prime} B^{\prime}} \\
& \left\langle 0\left|O_{c}^{b}\right| 0\right\rangle=2 \sin ^{2} \theta_{W} \delta_{\tilde{A} \tilde{B}}\left\{1+\frac{\alpha_{W}}{\pi}\left(\frac{\beta_{0}}{2}+2 \int_{z_{\text {cut }}}^{1} d z P_{g g}^{*}(z)\right) \log \left(\frac{\mu}{M_{W}}\right)\right\}
\end{aligned}
$$

Since our operators mix, we work with a matrix of anomalous dimensions,

$$
\begin{aligned}
\mu \frac{d}{d \mu}\left(\begin{array}{c}
O_{a}^{c, s} \\
O_{b}^{c, s}
\end{array}\right) & =\left(\begin{array}{cc}
\gamma_{\mu, a a}^{c, s} & \gamma_{\mu, a b}^{c, s} \\
\gamma_{\mu, b a}^{c, s} & \gamma_{\mu, b b}^{c, s}
\end{array}\right)\left(\begin{array}{c}
O_{a}^{c, s} \\
O_{b}^{c, s}
\end{array}\right), \\
\nu \frac{d}{d \nu}\left(\begin{array}{c}
O_{a}^{c, s} \\
O_{b}^{c, s}
\end{array}\right) & =\left(\begin{array}{cc}
\gamma_{\nu, a a}^{c, s} & \gamma_{\nu, a b}^{c, s} \\
\gamma_{\nu, b a}^{c, s} & \gamma_{\nu, b b}^{c, s}
\end{array}\right)\left(\begin{array}{c}
O_{a}^{c, s} \\
O_{b}^{c, s}
\end{array}\right),
\end{aligned}
$$

given by

$$
\begin{aligned}
\gamma_{\mu, a a}^{c} & =\frac{3 \alpha_{W}}{\pi} \log \left(\frac{\nu^{2}}{4 M_{\chi}^{2}}\right)+\frac{\alpha_{W}}{2 \pi}\left(\beta_{0}-2 \int_{z_{\mathrm{cut}}}^{1} d z P_{g g}^{*}(z)\right), \\
\gamma_{\mu, a b}^{c} & =-\frac{\alpha_{W}}{\pi} \log \left(\frac{\nu^{2}}{4 M_{\chi}^{2}}\right)+\frac{\alpha_{W}}{\pi} \int_{z_{\mathrm{cut}}}^{1} d z P_{g g}^{*}(z), \\
\gamma_{\mu, a a}^{s} & =-\frac{3 \alpha_{W}}{\pi} \log \left(\frac{\nu^{2}}{\mu^{2}}\right)+\frac{3 \alpha_{W}}{\pi}, \quad \gamma_{\mu, a b}^{s}=\frac{\alpha_{W}}{\pi} \log \left(\frac{\nu^{2}}{\mu^{2}}\right)-\frac{\alpha_{W}}{\pi}, \\
\gamma_{\mu, b b}^{c} & =\frac{\alpha_{W}}{\pi}\left(\frac{\beta_{0}}{2}+2 \int_{z_{\mathrm{cut}}}^{1} d z P_{g g}^{*}(z)\right) .
\end{aligned}
$$


Although they will play no further role in our calculation as our soft and collinear logs are minimized by running $\mu$ to $M_{W}$, for completeness we include our $\nu$-anomalous dimensions,

$$
\begin{array}{ll}
\gamma_{\nu, a a}^{c}=\frac{3 \alpha_{W}}{\pi} \log \left(\frac{\mu^{2}}{M_{W}^{2}}\right), & \gamma_{\nu, a a}^{s}=-\frac{3 \alpha_{W}}{\pi} \log \left(\frac{\mu^{2}}{M_{W}^{2}}\right), \\
\gamma_{\nu, a b}^{c}=-\frac{\alpha_{W}}{\pi} \log \left(\frac{\mu^{2}}{M_{W}^{2}}\right), \quad \gamma_{\nu, a b}^{s}=\frac{\alpha_{W}}{\pi} \log \left(\frac{\mu^{2}}{M_{W}^{2}}\right) .
\end{array}
$$

The terms in the RG matrix which are not explicitly stated are all 0.

\subsection{Resummed wino annihilation rate}

We use the RG invariance of the cross section to obtain equations for the Wilson coefficients of the operators in eq. (2.1),

$$
\begin{aligned}
& \mu \frac{d}{d \mu} C_{2,4}(\mu)=-\left(\gamma_{\mu, a a}^{c}+\gamma_{\mu, a a}^{s}\right) C_{2,4} \\
& \mu \frac{d}{d \mu} C_{1,3}(\mu)=-\left(\gamma_{\mu, b a}^{c}+\gamma_{\mu, b a}^{s}\right) C_{2,4}-\gamma_{\mu, b b}^{c} C_{1,3} .
\end{aligned}
$$

As discussed above, since the running of the Wilson coefficients combines both soft and collinear sectors, it must be independent of the rapidity scale $\nu$. Plugging in the results of eq. (2.12), we see that this is the case. The soft and collinear sectors have no large logs if we choose the $(\mu, \nu)$ scales to be $\left(M_{W}, M_{W}\right)$ and $\left(M_{\chi}, M_{W}\right)$, respectively. At leading double log accuracy we can resum all of the relevant terms by choosing $\mu=M_{W}$. In this case all the large logs reside in the renormalized parameter $C_{i}\left(\mu=M_{W}\right)$ and the rapidity running may be neglected.

Our objective is to perform a controlled calculation of the WIMP annihilation rate, including the important nonperturbative effects of Sommerfeld enhancement and Sudakov resummation. In our earlier papers, we focused on leading log (LL) resummation since at the thermal relic mass of $3 \mathrm{TeV}, \alpha_{W} \log ^{2}\left(\frac{2 M_{\chi}}{M_{W}}\right) \approx 0.6$, and over the range of the HESS experiment (up to around $18 \mathrm{TeV}$ ), this quantity grows larger than $1[7,8]$. Over the range probed by HESS, single-log corrections, $\alpha_{W} \log \left(\frac{2 M_{\chi}}{M_{W}}\right) \sim 10-20 \%$, which is non-negligible. Thus, we include them at fixed order, giving our LL' result. Therefore, we are accounting for all the terms of the form $\alpha_{W}^{n+1} \log ^{2 n+1}\left(\frac{2 M_{\chi}}{M_{W}}\right)$. To do a full NLL resummation, one would need to include the two-loop cusp anomalous dimension as well. Given the percent-scale effect this has, this is beyond the order to which we are working.

We can easily write down the solutions using the boundary conditions $C_{2}\left(2 M_{\chi}\right)=$ $-2 C_{1}\left(2 M_{\chi}\right), C_{4}\left(2 M_{\chi}\right)=C_{1}\left(2 M_{\chi}\right), C_{3}\left(2 M_{\chi}\right)=0[7,8]$,

$$
\begin{aligned}
& C_{1}(\mu)=\frac{1}{3} C_{1}\left(2 M_{\chi}\right)\left[\left(1+P_{g} L\right)+2 E_{1}\left(1+P_{g}^{\prime} L-\frac{\alpha_{W}^{2}}{\pi^{2}} \beta_{0} L^{3}\right)\right] \\
& C_{2}(\mu)=-2 C_{1}\left(2 M_{\chi}\right) E_{1}\left(1+P_{g}^{\prime} L-\frac{\alpha_{W}^{2}}{\pi^{2}} \beta_{0} L^{3}\right) \\
& C_{3}(\mu)=\frac{1}{3} C_{1}\left(2 M_{\chi}\right)\left[\left(1+P_{g} L\right)-E_{1}\left(1+P_{g}^{\prime} L-\frac{\alpha_{W}^{2}}{\pi^{2}} \beta_{0} L^{3}\right)\right] \\
& C_{4}(\mu)=C_{1}\left(2 M_{\chi}\right) E_{1}\left(1+P_{g}^{\prime} L-\frac{\alpha_{W}^{2}}{\pi^{2}} \beta_{0} L^{3}\right)
\end{aligned}
$$


where

$$
\begin{array}{rlrl}
P_{g} & =\frac{\alpha_{W}}{\pi}\left(\frac{\beta_{0}}{2}+2 \int_{z_{\mathrm{cut}}}^{1} d z P_{g g}^{*}(z)\right), & P_{g}^{\prime}=\frac{\alpha_{W}}{\pi}\left(\frac{\beta_{0}}{2}-\int_{z_{\mathrm{cut}}}^{1} d z P_{g g}^{*}(z)+3\right) \\
E_{1}=\exp \left[-\frac{3 \alpha_{W}}{\pi} \log ^{2}\left(\frac{2 M_{\chi}}{M_{W}}\right)\right], & L=\log \left(\frac{2 M_{\chi}}{M_{W}}\right) .
\end{array}
$$

To write down the final cross section, we need to evaluate the photon fragmentation function, eq. (2.5), at the scale $M_{W}$. At LL', the photon can fragment into charged SM states, so $F_{\tilde{A} \tilde{B}}^{\gamma}\left(M_{W}\right)$ is no longer its tree level value. In particular, this correction enters via the gauge-boson wavefunction renormalization, proportional to the corresponding $\beta$ function coefficient. Since this includes light fermionic states with mass lower than $M_{W}$, we therefore modify our result to take into account these corrections in the following way

$$
\frac{\alpha_{W}}{2 \pi} \beta_{0} \log \left(\frac{2 M_{\chi}}{M_{W}}\right) \rightarrow \frac{\alpha_{W}}{2 \pi} \beta_{0} \log \left(\frac{2 M_{\chi}}{M_{W}}\right)+\Pi_{\gamma \gamma},
$$

and similarly for hypercharge, where $\Pi_{\gamma \gamma}\left(\equiv \frac{\alpha(0)}{\alpha\left(M_{Z}\right)}-1\right)$ is the photon self energy function evaluated at the scale $M_{Z}$ and $\alpha$ is the fine-structure constant. The value of this quantity is derived from experiment to be -0.0594 [33]. We refer the reader to appendix C for details.

Following the discussion in [8], which fixes the tree-level matching coefficient ${ }^{4}$ as

$$
C_{1}\left(M_{\chi}\right)=\frac{\pi \alpha_{W}^{2} \sin ^{2} \theta_{W}}{2 M_{\chi}^{3}},
$$

we write the final cross-section in terms of the Sommerfeld enhancement factors,

$$
\begin{aligned}
\sigma v= & \frac{\pi \alpha_{W}^{2} \sin ^{2} \theta_{W}}{8 M_{\chi}^{4}}\left\{\frac{4}{3} f_{-}^{\prime}\left|\psi_{00}(0)\right|^{2}+4 f_{+}^{\prime}\left|\psi_{ \pm}(0)\right|^{2}\right. \\
& \left.+\frac{4}{3} f_{-}^{\prime}\left(\psi_{00}(0) \psi_{ \pm}^{*}(0)+\text { h.c. }\right)\right\},
\end{aligned}
$$

where

$$
\begin{aligned}
f_{ \pm}^{\prime}= & \left(1 \pm E_{1}\right)+\left(L P_{g}+\Pi_{\gamma \gamma}\right) \pm E_{1}\left(P_{g}^{\prime} L+\Pi_{\gamma \gamma}\right) \\
& \mp 2 E_{1} \frac{\alpha_{W}}{\pi} L^{2}\left(\frac{\alpha_{W}}{2 \pi} \beta_{0} L+\Pi_{\gamma \gamma}\right) .
\end{aligned}
$$

We define the nonrelativistic wavefunctions as

$$
\begin{aligned}
& \psi_{00}(0)=\left\langle 0\left|\left(\chi^{0}\right)^{\top} i \sigma^{2} \chi^{0}\right| \chi^{0} \chi^{0}\right\rangle_{S}=2 \sqrt{2} M_{\chi} \psi_{1}(0) \\
& \psi_{ \pm}(0)=\left\langle 0\left|\left(\chi^{-}\right)^{\top} i \sigma^{2} \chi^{+}\right| \chi^{0} \chi^{0}\right\rangle_{S}=2 M_{\chi} \psi_{2}(0),
\end{aligned}
$$

where $\left|\chi^{0} \chi^{0}\right\rangle_{S}=\frac{1}{\sqrt{2}}\left(\left|\chi_{\uparrow}^{0}\left(p_{1}\right) \chi_{\downarrow}^{0}\left(p_{2}\right)\right\rangle-\left|\chi_{\downarrow}^{0}\left(p_{1}\right) \chi_{\uparrow}^{0}\left(p_{2}\right)\right\rangle\right)$, a spin singlet state. The factors $\psi_{1,2}$ in eq. (2.21) are the dimensionless Sommerfeld enhancement factors. We briefly sketch their calculation in appendix $\mathrm{B}$, with more details in $[5,8,23]$. We note that the $\mathrm{LL}^{\prime}$ rate is schematically the same as LL, the only difference coming from the correction to the Sudakov factors in eq. (2.20). We recover the earlier result by setting $L, \Pi_{\gamma \gamma}$ to 0 in that formula.

\footnotetext{
${ }^{4}$ The Wilson coefficients in eq. (2.4) contained factors of $\delta\left(2 M_{\chi}-n \cdot p\right)$, where $p$ is the total momentum in the $n$-direction, but our convention in [8] performed the integral over the $\delta$-constrained variable in calculating $C_{1}$ and the other coefficients related to it.
} 


\section{Higgsino}

We next turn to the case of higgsino-like dark matter. Just as with the wino, one can take this state to be the neutralino LSP, in the limit where mixing with gauginos is negligible. However, it could arise in a simplified model of Dark Matter, where we augment the SM by two $\mathrm{SU}(2)$ doublets with hypercharge $\pm \frac{1}{2}$. The one complication in this sector is that direct detection constraints prevent the DM to from being a pure doublet [24]. We need mixing with another state (such as the gauginos) to split the Majorana components of the neutral fermion by at least $200 \mathrm{keV}$. However, the requirements on the state being mixed are only that it have mass less than $10^{9} \mathrm{GeV}$, which is sufficiently decoupled that in practice we work with a doublet possessing splittings between its neutral components and between the neutral and charged fermions. These mass differences are much more model-dependent than that of the wino. We thus study both a narrowly-split limit, just allowed by direct detection constraints, and a more widely split scenario which could arise in the MSSM neutralino sector from having gaugino soft masses $M_{1,2}$ a factor of a few greater than $|\mu|$.

Prior to dressing with soft Wilson-lines, the minimal operator basis that we need is

$$
\begin{aligned}
& O_{1}=g^{2} g^{\prime 2}\left[\left(\bar{\chi} \gamma^{5} \tau^{a} \chi\right)\left(\bar{\chi} \gamma^{5} \tau^{a} \chi\right)+\frac{\tan \theta_{W}^{2}}{4}\left(\bar{\chi} \gamma^{5} \chi\right)\left(\bar{\chi} \gamma^{5} \chi\right)\right] B B \\
& O_{2}=\frac{g^{4}}{4}\left(\bar{\chi} \gamma^{5} \chi\right)\left(\bar{\chi} \gamma^{5} \chi\right) B^{A} B^{A} \\
& O_{3}=g^{2} g^{\prime 2}\left(\bar{\chi} \gamma^{5} \tau^{A} \chi\right)\left(\bar{\chi} \gamma^{5} \tau^{B} \chi\right) B^{A} B^{B} \\
& O_{4}=\left(\frac{g^{3} g^{\prime}+g g^{\prime 3}}{2}\right)\left[\left(\bar{\chi} \gamma^{5} \tau^{A} \chi\right)\left(\bar{\chi} \gamma^{5} \chi\right) B^{A} B+\left(\bar{\chi} \gamma^{5} \chi\right)\left(\bar{\chi} \gamma^{5} \tau^{A} \chi\right) B B^{A}\right] \\
& O_{5}=\left(\bar{\chi} \gamma^{5} \tau^{A} \chi\right)\left(\bar{\chi} \gamma^{5} \tau^{A} \chi\right) B^{B} B^{B} .
\end{aligned}
$$

$B^{A}$ is the $\mathrm{SU}(2)$ gauge-boson field (implicitly the $\perp$ portion of $B_{n}^{A}$ ) while $B_{n}$ is the hypercharge field. The coupling-dependent prefactors arise from matching the $2 \rightarrow 2$ WIMP annihilation to gauge bosons at tree-level. This gives dimension-five, $(\bar{\chi} \chi) B B$ operators, with which we then perform an OPE (cf. figure 1) to obtain operators for the semi-inclusive annihilation rate to $\gamma+X$. Normalizing the operators in this way automatically gives 0 when we add up $O_{1-4}\left(O_{5}\right.$ is not generated at tree level), as it should, when we set the fermions to their neutral components and project the gauge bosons onto photons, $B^{A} \rightarrow \sin \theta_{W} \gamma$, $B \rightarrow \cos \theta_{W} \gamma$. Thus, just as in the wino sector, we will just need a nonzero tree-level process, like $\chi^{+} \chi^{-} \rightarrow \gamma \gamma+\frac{1}{2} \gamma Z$, to fix a common Wilson coefficient. ${ }^{5}$ As explained below eq. (2.1), we use the vacuum insertion approximation in the WIMP sector, but we have dropped the explicit vacuum projector. Just as before, there is also an implicit Lorentz contraction and projection onto a single-photon state between the $B, B^{A}$ fields (cf. eq. (2.2)). As in the wino case, since we are annihilating Majorana fermions, the only relevant bilinear is $\bar{\chi} \gamma_{5} \chi$. The spin-one operators are irrelevant since Fermi statistics would lead to an antisymmetric $\mathrm{SU}(2)$ initial state, and we are interested in the annihilation of two neutral

\footnotetext{
${ }^{5}$ Because of the identity $\tau_{i j}^{a} \tau_{i^{\prime} j^{\prime}}^{a}=\frac{1}{2} \delta_{i j^{\prime}} \delta_{i^{\prime} j}-\frac{1}{4} \delta_{i j} \delta_{i^{\prime} j^{\prime}}$, the basis in eq. (3.1) is not orthogonal. For simplicity though, we have chosen to contract color indices between fermions on the same side of the cut.
} 
particles. Furthermore, P-wave annihilation is velocity suppressed. In the fermion sector we have combined the two doublets into $\chi$ with,

$$
\chi \equiv\left(\begin{array}{c}
\tilde{h}_{u} \\
\epsilon \tilde{h}_{d}^{*}
\end{array}\right), \quad \bar{\chi}=\left(\begin{array}{lll}
-\epsilon \tilde{h}_{d} & \tilde{h}_{u}^{*}
\end{array}\right),
$$

where $\tilde{h}_{u, d}$ are the higgsino gauge eigenstates. Our operators therefore also have an intrinsic identity in doublet-space. In the limit where the mass eigenstates are pure higgsino, we have

$$
\begin{aligned}
& \chi_{1}^{0}=\frac{1}{\sqrt{2}}\left(h_{d}^{0}-h_{u}^{0}\right) \\
& \chi_{2}^{0}=-\frac{i}{\sqrt{2}}\left(h_{d}^{0}+h_{u}^{0}\right),
\end{aligned}
$$

where $\chi_{1}^{0}$ is the lighter mass state. We also have a charged Dirac fermion, $\chi^{+\top}=\left(\tilde{h}_{u}^{+} \tilde{h}_{d}^{-*}\right)$.

We can now decompose the operators in eq. (3.1) into the soft and collinear sectors, as in eq. (2.3), obtaining a factorization theorem for the annihilation rate. This is identical to our approach with winos and some of the resulting operators will even be identical, therefore receiving the same radiative corrections. In the soft sector we get,

$$
\begin{aligned}
O_{s}^{a} & =S_{v A^{\prime} A}^{\top} S_{v B B^{\prime}} S_{n \tilde{A} A}^{\top} S_{n B \tilde{B}} \\
O_{s}^{b} & =\delta_{\tilde{A} \tilde{B}} \delta_{A^{\prime} B^{\prime}} \\
O_{s}^{c} & =S_{v A^{\prime} A}^{\top} S_{n \tilde{A} A^{\top}}^{\top} .
\end{aligned}
$$

In terms of the basis in eq. (3.1), these operators dress $O_{3}, O_{5}$, and $O_{4}$, respectively. We see that $O_{s}^{a}$ and $O_{s}^{b}$ are identical to operators found in the wino sector and $O_{s}^{c}$ is effectively the "square root" of $O_{s}^{a}$. The full operators $O_{1}$ and $O_{2}$ contain various singlet structures, some of which are trivial in triplet-space, but they do not receive radiative corrections and are not generated by mixing, so do we not detail their form. Despite the higgsinos being doublets, we can obtain identical soft Wilson-line structures to the wino case by using the identity $s^{\dagger} \tau^{a} s=\tau^{c}\left(S^{\top}\right)^{c a}$, where $s$ is the soft Wilson-line in the fundamental representation and $S$ is the adjoint. In the collinear sector, we have

$$
\begin{aligned}
O_{c}^{a} & =B_{\tilde{A}} B_{\tilde{B}} \\
O_{c}^{b} & =B_{D} B_{D} \delta_{\tilde{A} \tilde{B}} . \\
O_{c}^{c} & =B_{\tilde{A}} B+B B_{\tilde{A}} \\
O_{c}^{d} & =B B
\end{aligned}
$$

where here $O_{c}^{a, b}$ are repeats from the wino sector. ${ }^{6}$

The details of our factorization theorem, which is identical to the wino result, can be found in $[7,8]$. Because of the more involved operator structure, we will also decompose

\footnotetext{
${ }^{6}$ Strictly speaking, $O_{2}$ in eq. (3.1) contains a fifth collinear structure, which we could call $O_{c}^{e}=B_{D} B_{D}$. It has no additional $\delta$, unlike $O_{c}^{b}$, since the rest of the operator has trivial triplet structure. The loop corrections to these operators are identical, up to the overall $\delta$. We can therefore recycle our $O_{c}^{b}$ calculation for $O_{c}^{e}$, and neither generates other structures. The running of $O_{c}^{a}$ though, will generate contributions to $O_{c}^{b}$, but not $O_{c}^{e}$.
} 
the operators in eq. (3.1) into the WIMP sector, defining $O_{i}^{\mathrm{NR}}$ to be the $\chi$-field component of the full operator $O_{i}$, including any Dirac and color matrices that contract with the fermions. For example,

$$
O_{3 \mathrm{NR}}^{A B}=\left(\bar{\chi} \gamma^{5} \tau^{A} \chi\right)\left(\bar{\chi} \gamma^{5} \tau^{B} \chi\right)
$$

The annihilation spectrum may be written as

$$
\begin{aligned}
\frac{1}{E_{\gamma}} \frac{d \sigma}{d E_{\gamma}}= & \frac{1}{4 M_{\chi}^{2} v}\left\{\left\langle 0\left|O_{s}^{a}\right| 0\right\rangle\left[\int d n \cdot p\left\{C_{3}\left(M_{\chi}, n \cdot p\right)\left\langle p_{1} p_{2}\left|O_{3 \mathrm{NR}}^{A^{\prime} B^{\prime}}\right| p_{1} p_{2}\right\rangle\right\} F_{\tilde{A} \tilde{B}}^{\gamma}\left(\frac{2 E_{\gamma}}{n \cdot p}\right)\right]\right. \\
& +\left[\int d n \cdot p \left\{C_{2}\left(M_{\chi}, n \cdot p\right)\left\langle p_{1} p_{2}\left|O_{2 \mathrm{NR}}\right| p_{1} p_{2}\right\rangle\right.\right. \\
& \left.\left.+C_{5}\left(M_{\chi}, n \cdot p\right)\left\langle p_{1} p_{2}\left|O_{5 \mathrm{NR}}\right| p_{1} p_{2}\right\rangle\right\} F_{\tilde{A} \tilde{A}}^{\gamma}\left(\frac{2 E_{\gamma}}{n \cdot p}\right)\right] \\
& +\left\langle 0\left|O_{s}^{c}\right| 0\right\rangle\left[\int d n \cdot p\left\{C_{4}\left(M_{\chi}, n \cdot p\right)\left\langle p_{1} p_{2}\left|O_{4 \mathrm{NR}}^{A^{\prime}}\right| p_{1} p_{2}\right\rangle\right\} F_{\tilde{A}}^{\gamma}\left(\frac{2 E_{\gamma}}{n \cdot p}\right)\right] \\
& \left.+\left[\int d n \cdot p\left\{C_{1}\left(M_{\chi}, n \cdot p\right)\left\langle p_{1} p_{2}\left|O_{1 \mathrm{NR}}\right| p_{1} p_{2}\right\rangle\right\} F^{\gamma}\left(\frac{2 E_{\gamma}}{n \cdot p}\right)\right]\right\}
\end{aligned}
$$

where $F_{\tilde{A} \tilde{B}}^{\gamma}, F_{\tilde{A}}^{\gamma}, F^{\gamma}$ are fragmentation functions defined by

$$
\begin{aligned}
F_{\tilde{A} \tilde{B}}^{\gamma}\left(\frac{n \cdot k}{n \cdot p}\right)= & \int \frac{d x_{-}}{2 \pi} e^{i n \cdot p x_{-}}\left\langle 0\left|B_{n, \tilde{A}}^{\perp \mu}\left(x_{-}\right)\right| \gamma\left(k_{n}\right)+X_{n}\right\rangle \\
& \times\left\langle\gamma\left(k_{n}\right)+X_{n}\left|B_{n, \mu \tilde{B}}^{\perp}(0)\right| 0\right\rangle, \\
F_{\tilde{A}}^{\gamma}\left(\frac{n \cdot k}{n \cdot p}\right)= & \int \frac{d x_{-}}{2 \pi} e^{i n \cdot p x_{-}}\left\langle 0\left|B_{n, \tilde{A}}^{\perp \mu}\left(x_{-}\right)\right| \gamma\left(k_{n}\right)+X_{n}\right\rangle, \\
& \times\left\langle\gamma\left(k_{n}\right)+X_{n}\left|B_{n, \mu}^{\perp}(0)\right| 0\right\rangle, \\
F^{\gamma}\left(\frac{n \cdot k}{n \cdot p}\right)= & \int \frac{d x_{-}}{2 \pi} e^{i n \cdot p x_{-}}\left\langle 0\left|B_{n}^{\perp \mu}\left(x_{-}\right)\right| \gamma\left(k_{n}\right)+X_{n}\right\rangle \\
& \times\left\langle\gamma\left(k_{n}\right)+X_{n}\left|B_{n, \mu}^{\perp}(0)\right| 0\right\rangle .
\end{aligned}
$$

$C_{1-4}$ are the matching coefficients that give the probability for the dark matter to annihilate with total +-momentum in the $n$-direction of $n \cdot p$. As in eq. (2.4), they contain $n$-component of the expanded, overall $\delta$-function for the process, constraining $n \cdot p=2 M_{\chi}$. $F^{\gamma}$ and $F_{\tilde{A} \tilde{A}}^{\gamma}$ are the canonical fragmentation functions giving the probability of an initial boson with momentum $p$ to yield a photon with momentum fraction $n \cdot k / n \cdot p$ after splitting. Just as with the wino, we get gauge nonsinglet fragmentation functions, which are rendered physical by electroweak symmetry breaking. Since the contributions in eq. (3.8) proportional to $F_{\tilde{A} \tilde{A}}^{\gamma}$ are not sensitive to the nonsinglet nature of the initial state, they will only contribute large double logs from mixing with $\mathrm{O}_{3}$.

\subsection{Anomalous dimensions}

We now calculate the anomalous dimensions for the soft and collinear sector operators in eqs. (3.5), (3.6). Four of the operators $O_{c, s}^{a}, O_{c, s}^{b}$ are exactly the same as the wino case. 


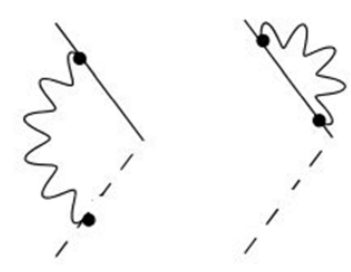

Figure 4. The diagrams contributing to $O_{s}^{c}$. The solid/dashed lines indicate time/light-like Wilson lines.
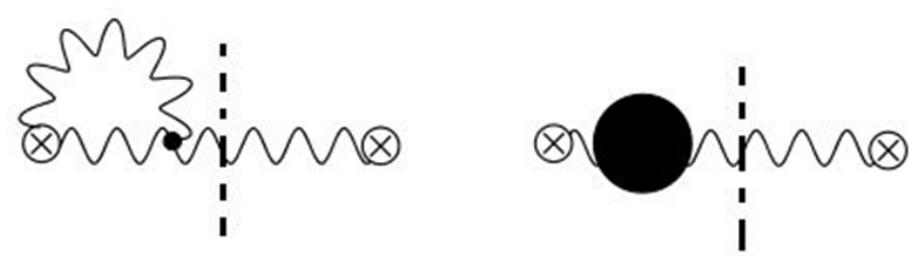

Figure 5. The diagrams contributing to $O_{c}^{c}$. The dark blob contains fermion, Higgs and gauge boson loops.

However, due to the coupling of the higgsino to the Hypercharge field, we obtain three new nontrivial structures, $O_{c, s}^{c}$, contained in $O_{3}$, and $O_{d}^{c}$ in $O_{4}$, which we repeat here,

$$
\begin{aligned}
O_{s}^{c} & =S_{v A^{\prime} A^{\top}}^{\top} S_{n \tilde{A} A}^{\top}, \\
O_{c}^{c} & =B_{\tilde{A}} B+B B_{\tilde{A}}, \\
O_{c}^{d} & =B B .
\end{aligned}
$$

The operator $O_{s}^{c}$ has a unique color structure, so that it mixes only into itself (figure 4). $O_{c}^{c}$ contributes to a hybrid Weak-Hypercharge fragmentation function, which is why it gets only virtual corrections (figure 5). The hypercharge field is Abelian, so it does not get any Wilson-line contributions.

$$
\begin{aligned}
\left\langle 0\left|O_{s}^{c}\right| 0\right\rangle= & \delta_{A^{\prime} \tilde{A}}\left[1-\frac{\alpha_{W}}{\pi}\left\{2 \log \left(\frac{\nu}{\mu}\right) \log \left(\frac{\mu}{M_{W}}\right)+\log ^{2}\left(\frac{\mu}{M_{W}}\right)-\log \left(\frac{\mu}{M_{W}}\right)\right\}\right] \\
\left\langle 0\left|O_{c}^{c}\right| 0\right\rangle= & 2 \sin \theta_{W} \cos \theta_{W} \delta_{\tilde{A} 3}\left[1-\frac{\alpha_{W}}{\pi}\left\{2 \log \left(\frac{M_{\chi}}{\nu}\right) \log \left(\frac{\mu}{M_{W}}\right)\right\}\right. \\
& \left.+\frac{\alpha_{W}}{4 \pi} \beta_{0} \log \left(\frac{\mu}{M_{W}}\right)+\frac{\alpha^{\prime}}{4 \pi} \beta_{0}^{\prime} \log \left(\frac{\mu}{M_{W}}\right)\right]
\end{aligned}
$$

where $\beta_{0}^{\prime}=-\frac{41}{6}$ is the leading order hypercharge beta function coefficient. $O_{c}^{d}$, which contributes to the hypercharge fragmentation function, only gets wavefunction renormalization corrections (figure 6), which we discuss further in appendix C.

$$
\left\langle 0\left|O_{c}^{d}\right| 0\right\rangle=2 \cos ^{2} \theta_{W}\left[1+\frac{\alpha^{\prime}}{2 \pi} \beta_{0}^{\prime} \log \left(\frac{\mu}{M_{W}}\right)\right]
$$




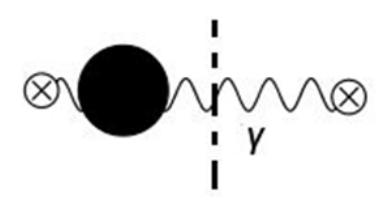

Figure 6. The diagrams contributing to $O_{c}^{d}$. The dark blob contains fermion and Higgs loops.

From these matrix elements, we can compute the regular and rapidity anomalous dimensions. None of the new contributions generates any mixing, so we simply get,

$$
\begin{aligned}
\mu \frac{d}{d \mu} O_{c}^{c} & =\gamma_{\mu c}^{c} O_{c}^{c}, \quad \nu \frac{d}{d \nu} O_{c}^{c}=\gamma_{\nu c}^{c} O_{c}^{c} \\
\mu \frac{d}{d \mu} O_{s}^{c} & =\gamma_{\mu c}^{s} O_{s}^{c}, \quad \nu \frac{d}{d \nu} O_{s}^{c}=\gamma_{\nu c}^{s} O_{s} \\
\mu \frac{d}{d \mu} O_{c}^{d} & =\gamma_{\mu d}^{c} O_{c}^{d}
\end{aligned}
$$

and

$$
\begin{array}{rlrl}
\gamma_{\mu c}^{c} & =\frac{\alpha_{W}}{\pi}\left(\log \left(\frac{\nu^{2}}{4 M_{\chi}^{2}}\right)+\frac{\beta_{0}}{4}\right)+\frac{\alpha^{\prime}}{4 \pi} \beta_{0}^{\prime}, & \gamma_{\nu c}^{c}=\frac{\alpha_{W}}{\pi} \log \left(\frac{\mu^{2}}{M_{W}^{2}}\right) \\
\gamma_{\mu c}^{s}=-\frac{\alpha_{W}}{\pi} \log \left(\frac{\nu^{2}}{\mu^{2}}\right)+\frac{\alpha_{W}}{\pi}, & \gamma_{\nu c}^{s}=-\frac{\alpha_{W}}{\pi} \log \left(\frac{\mu^{2}}{M_{W}^{2}}\right) \\
\gamma_{\mu d}^{c}=\frac{\alpha^{\prime}}{2 \pi} \beta_{0}^{\prime} &
\end{array}
$$

The terms not explicitly stated are all 0 . We note that $O_{c}^{d}$ has no rapidity anomalous dimension as it receives contributions only at the single-log level. We trivially see that $\nu$ dependence drops out, as it must, when we add $\gamma_{\mu c}^{c}+\gamma_{\mu c}^{s}$, the combination that will appear in running the Wilson coefficient, $C_{4}$, for the operator in eq. (3.1). Also, we see that both soft and collinear rapidity anomalous dimensions will vanish when we set $\mu=m_{W}$, so we will not need the rapidity RG.

\subsection{Resummed higgsino annihilation rate}

We can now use RG invariance of the cross section to compute the RG equations for the Wilson coefficients

$$
\begin{aligned}
\mu \frac{d}{d \mu} C_{1} & =-\gamma_{\mu d}^{c} C_{1} \\
\mu \frac{d}{d \mu} C_{2} & =-\gamma_{\mu, b b}^{c} C_{2} \\
\mu \frac{d}{d \mu} C_{3} & =-\left(\gamma_{\mu, a a}^{c}+\gamma_{\mu, a a}^{s}\right) C_{3} \\
\mu \frac{d}{d \mu} C_{4} & =-\left(\gamma_{\mu c}^{c}+\gamma_{\mu c}^{s}\right) C_{4} \\
\mu \frac{d}{d \mu} C_{5} & =-\frac{1}{3}\left(\gamma_{\mu, a b}^{c}+\gamma_{\mu, a b}^{s}\right) C_{3}-\gamma_{\mu, b b}^{c} C_{5}
\end{aligned}
$$


As in the wino case, our objective is to obtain the LL' cross section in order to have a controlled calculation for higgsino annihilation. For the thermal relic mass of $1 \mathrm{TeV}$, we see that the Sudakov logarithm is not so $\operatorname{large}, \log \left(2 M_{\chi} / M_{W}\right) \approx 3.2$, so a pure LL calculation at this value may not capture all important contributions. Tree-level matching tells us $C_{1}\left(2 M_{\chi}\right)=C_{2-4}\left(2 M_{\chi}\right) \equiv C\left(2 M_{\chi}\right)$ and $C_{5}\left(2 M_{\chi}\right)=0$. Using these boundary conditions and the RG equations, we can write down the solutions for the Wilson coefficients at this order,

$$
\begin{aligned}
& C_{1}(\mu)=C\left(2 M_{\chi}\right)\left[1+\frac{\alpha^{\prime}}{2 \pi} \beta_{0}^{\prime} L\right] \\
& C_{2}(\mu)=C\left(2 M_{\chi}\right)\left[1+P_{g} L\right] \\
& C_{3}(\mu)=C\left(2 M_{\chi}\right) E_{1}\left[1+P_{g}^{\prime} L-\frac{\alpha_{W}^{2}}{\pi^{2}} \beta_{0} L^{3}\right] \\
& C_{4}(\mu)=C\left(2 M_{\chi}\right) E_{2}\left[1+\left(\frac{\alpha_{W}}{4 \pi}\left(4+\beta_{0}\right)+\frac{\alpha^{\prime}}{4 \pi} \beta_{0}^{\prime}\right) L-\frac{\alpha_{W}^{2}}{3 \pi^{2}} \beta_{0} L^{3}\right] \\
& C_{5}(\mu)=C\left(2 M_{\chi}\right)\left[\frac{1}{3}\left(1+P_{g} L\right)-\frac{1}{3} E_{1}\left(1+P_{g}^{\prime} L-\frac{\alpha_{W}^{2}}{\pi^{2}} \beta_{0} L^{3}\right)\right],
\end{aligned}
$$

where we use the same functions, as in eq. (2.16), which we repeat here, along with the new $E_{2}$,

$$
\begin{array}{ll}
P_{g}=\frac{\alpha_{W}}{\pi}\left(\frac{\beta_{0}}{2}+2 \int_{z_{\mathrm{cut}}}^{1} d z P_{g g}^{*}(z)\right), & P_{g}^{\prime}=\frac{\alpha_{W}}{\pi}\left(\frac{\beta_{0}}{2}-\int_{z_{\mathrm{cut}}}^{1} d z P_{g g}^{*}(z)+3\right) \\
E_{1}=\exp \left[-\frac{3 \alpha_{W}}{\pi} \log ^{2}\left(\frac{2 M_{\chi}}{M_{W}}\right)\right], & L=\log \left(\frac{2 M_{\chi}}{M_{W}}\right) \\
E_{2}=\exp \left[-\frac{\alpha_{W}}{\pi} \log ^{2}\left(\frac{2 M_{\chi}}{M_{W}}\right)\right] . &
\end{array}
$$

The cross section can now be obtained by evaluating the effective theory matrix elements at their natural scale $\mu \sim M_{W}$.

In order to get a numerical result, we must perform the tree-level matching to determine $C\left(2 M_{\chi}\right)$, which we again do by computing $\chi^{+} \chi^{-} \rightarrow \gamma \gamma+\frac{1}{2} \gamma Z$, which at this order is $\chi^{+} \chi^{-} \rightarrow \gamma+X$. The leading order cross section is

$$
\sigma v=\frac{\pi \alpha_{W} \alpha^{\prime}}{4 M_{\chi}^{2}}
$$

For both this matching and our later determination of the neutral WIMP annihilation rate, it is useful to write our fermion bilinears in terms of mass eigenstates. For simplicity, we drop the $\gamma^{5}$ common to all terms in this calculation.

$$
\begin{aligned}
\bar{\chi} \chi & =\frac{1}{2} \bar{\chi}_{1}^{0} \chi_{1}^{0}+\frac{1}{2} \bar{\chi}_{2}^{0} \chi_{2}^{0}+\chi^{+} \chi^{-} \\
\bar{\chi} \tau^{3} \chi & =-\frac{1}{4} \bar{\chi}_{1}^{0} \chi_{1}^{0}-\frac{1}{4} \bar{\chi}_{2}^{0} \chi_{2}^{0}+\frac{1}{2} \chi^{+} \chi^{-} .
\end{aligned}
$$


From the effective theory description, we calculate this particular cross section from the basis of operators in eq. (3.1), projecting the fermions onto the charged state and the gauge bosons onto photons,

$$
\sigma v=\frac{1}{4 M_{\chi}^{2}} C\left(2 M_{\chi}\right){ }_{S}\left\langle\chi^{+} \chi^{-}\left|\sum_{i=1}^{4} O_{i}\right| \chi^{+} \chi^{-}\right\rangle_{S},
$$

The first term on the r.h.s. is the flux factor. Our conventions, including the polarization sum, fix

$$
{ }_{S}\left\langle\chi^{+} \chi^{-}\left|\sum_{i=1}^{4} O_{i}\right| \chi^{+} \chi^{-}\right\rangle_{S}=32 \pi^{2} \alpha_{W} \alpha^{\prime}\left|\left\langle 0\left|\chi^{+} \gamma^{5} \chi^{-}\right| \chi^{+} \chi^{-}\right\rangle_{S}\right|^{2}
$$

and at tree level, $\left\langle 0\left|\chi^{+} \gamma^{5} \chi^{-}\right| \chi^{+} \chi^{-}\right\rangle_{S}=2 \sqrt{2} M_{\chi}$. Thus, we get $^{7}$

$$
C\left(2 M_{\chi}\right)=\frac{1}{256 \pi M_{\chi}^{2}}
$$

where we have absorbed the bosonic phase space, for both observed photon and the recoil particle in $C\left(2 M_{\chi}\right)$.

We now need to account for the Sommerfeld enhancement of the higgsino annihilation rate. The details of the potential are given in appendix B. The computation is quite similar to the wino, but with an additional neutral, Majorana fermion. We thus get, in analogy with eq. (2.21),

$$
\begin{aligned}
& \psi_{00}^{1}(0)=\left\langle 0\left|\left(\chi_{1}^{0}\right)^{\top} i \sigma^{2} \chi_{1}^{0}\right| \chi_{1}^{0} \chi_{1}^{0}\right\rangle_{S}=2 \sqrt{2} M_{\chi} \psi_{1}(0) \\
& \psi_{00}^{2}(0)=\left\langle 0\left|\left(\chi_{2}^{0}\right)^{\top} i \sigma^{2} \chi_{2}^{0}\right| \chi_{1}^{0} \chi_{1}^{0}\right\rangle_{S}=2 \sqrt{2} M_{\chi} \psi_{2}(0) \\
& \psi_{ \pm}(0)=\left\langle 0\left|\left(\chi^{-}\right)^{\top} i \sigma^{2} \chi^{+}\right| \chi_{1}^{0} \chi_{1}^{0}\right\rangle_{S}=2 M_{\chi} \psi_{3}(0)
\end{aligned}
$$

where in addition to having an overlap between the asymptotic, $\left|\chi_{1}^{0} \chi_{1}^{0}\right\rangle_{S}$ state and annihilation of the chargino at the origin, captured by $\psi_{ \pm}$, we also account for the possibility that the short-distance annihilation occurs via the excited neutral state $\chi_{2}^{0}$. The functions $\psi_{1-3}(0)$ are the dimensionless Sommerfeld factors obtained from solving the potential in appendix B. ${ }^{8}$ We can now include the results of running the Wilson coefficients (eq. (3.16))

\footnotetext{
${ }^{7}$ Unlike the wino calculation, we did not pull out an overall factor of $M_{\chi}$ from $C\left(2 M_{\chi}\right)$, which is why this matching coefficient is dimension -2 , while in eq. (2.18) we get a quantity of dimension -3 . As with the wino though, in our explicit Wilson coefficients, we integrate the trivial $\delta$-functions, $\delta\left(2 M_{\chi}-n \cdot p\right)$ implicit in their definition in eq. (3.8).

${ }^{8}$ For consistency with the wino notation, we have written the wavefunctions in terms of two-component fermions. It is straightforward to convert back and forth between these and the four-component formalism we have used elsewhere.
} 
to get the annihilation rate for $\chi_{1}^{0} \chi_{1}^{0} \rightarrow \gamma+X$,

$$
\begin{aligned}
\sigma v= & \frac{\pi \alpha_{W} \alpha^{\prime}}{16 M_{\chi}^{4}}\left[\frac{1}{4}\left[\left|\psi_{00}^{1}(0)\right|^{2}+\left|\psi_{00}^{2}(0)\right|^{2}+\left(\psi_{00}^{1} \psi_{00}^{2 *}+\text { c.c. }\right)\right] \times\right. \\
& \left\{\left(1-E_{2}\right)\left\{1+\left(\frac{\alpha_{W}}{\pi}+\frac{\alpha_{W}}{4 \pi} \beta_{0}+\frac{\alpha^{\prime}}{4 \pi} \beta_{0}^{\prime}\right) L+\Pi_{\gamma \gamma}\right\}-\frac{s_{W}^{2}}{3}\left(1-E_{1}\right)\left(1+P_{g}^{\prime} L+\Pi_{\gamma \gamma}\right)\right. \\
& \left.-c_{W}^{2} \frac{\alpha_{W}}{\pi}\left\{1-\int_{z_{\text {cut }}}^{1} d z P_{g g}^{*}(z)\right\} L+\left(E_{2}-s_{W}^{2} E_{1}\right)\left(\frac{2 \alpha_{W}}{3 \pi}\right) L^{2}\left(\frac{\alpha_{W} \beta_{0}}{2 \pi} L+\Pi_{\gamma \gamma}\right)\right\} \\
& +\left|\psi_{ \pm}(0)\right|^{2} \times \\
& \left\{\left(1+E_{2}\right)\left\{1+\left(\frac{\alpha_{W}}{\pi}+\frac{\alpha_{W}}{4 \pi} \beta_{0}+\frac{\alpha^{\prime}}{4 \pi} \beta_{0}^{\prime}\right) L+\Pi_{\gamma \gamma}\right\}-\frac{s_{W}^{2}}{3}\left(1-E_{1}\right)\left(1+P_{g}^{\prime} L+\Pi_{\gamma \gamma}\right)\right. \\
& \left.-c_{W}^{2} \frac{\alpha_{W}}{\pi}\left\{1-\int_{z_{\mathrm{cut}}}^{1} d z P_{g g}^{*}(z)\right\} L-\left(E_{2}+s_{W}^{2} E_{1}\right)\left(\frac{2 \alpha_{W}}{3 \pi}\right) L^{2}\left(\frac{\alpha_{W} \beta_{0}}{2 \pi} L+\Pi_{\gamma \gamma}\right)\right\} \\
& +\frac{1}{2}\left(\psi_{00}^{1} \psi_{ \pm}^{*}+\psi_{00}^{2} \psi_{ \pm}^{*}+\text { c.c. }\right) \times \\
& \left\{\frac{s_{W}^{2}}{3}\left(1-E_{1}\right)\left(1+P_{g}^{\prime} L+\Pi_{\gamma \gamma}\right)-s_{W}^{2} \frac{\alpha_{W}}{\pi} L-\left(s_{W}^{2}-c_{W}^{2}\right)\left\{\frac{\alpha_{W}}{4 \pi} \beta_{0}-\frac{\alpha^{\prime}}{4 \pi} \beta_{0}^{\prime}\right\} L\right. \\
& \left.+c_{W}^{2} \frac{\alpha_{W}}{\pi}\left\{\int_{z_{\mathrm{cut}}}^{1} d z P_{g g}^{*}(z)\right\} L+s_{W}^{2} E_{1}\left(\frac{2 \alpha_{W}}{3 \pi}\right) L^{2}\left(\frac{\alpha_{W} \beta_{0}}{2 \pi} L+\Pi_{\gamma \gamma}\right)\right\},
\end{aligned}
$$

where $L=\log \left(\frac{2 M_{\chi}}{M_{W}}\right)$ and $P_{g}, E_{i}$, etc. are given in eq. (3.17).

As in the case of the wino, we must account for corrections to the photon fragmentation functions eq. (3.9) beyond tree level, including the possibility to split below the scale $M_{W}$. This is seen in the above equation (3.24) in the $\Pi_{\gamma \gamma}$ term, which, as defined earlier is the photon self energy function at the scale $M_{W}$. For details, see the discussion around eq. (2.17) and appendix C.

\section{Dark matter constraints and conclusion}

Having calculated tree level matching, LL' resummation, and computed the Sommerfeld enhancement numerically, we can now evaluate the differential cross section for $\chi^{0} \chi^{0} \rightarrow$ $\gamma+X$, given for winos (eq. (2.19)) and higgsinos (eq. (3.24)).

In our previous calculation at LL, we found the effect of higher order corrections to be very modest [8]. This is in contrast to those groups that performed an exclusive two-body calculation $[17,18]$. We therefore wanted to examine whether the single-log contributions might be a large factor, which prompted this calculation at LL'. As mentioned in the Introduction, working at single-log brings in new effects which have the potential to be large: 1) photon fragmentation to pairs of SM particles, 2) real emission processes with $z<z_{\text {cut }}$. As we now show, fragmentation is a minor effect, but accounting for finite energy fraction, $z$, is numerically important enough to warrant a follow-up study [20]. As before, we adapt the exclusion analysis of [18]. 


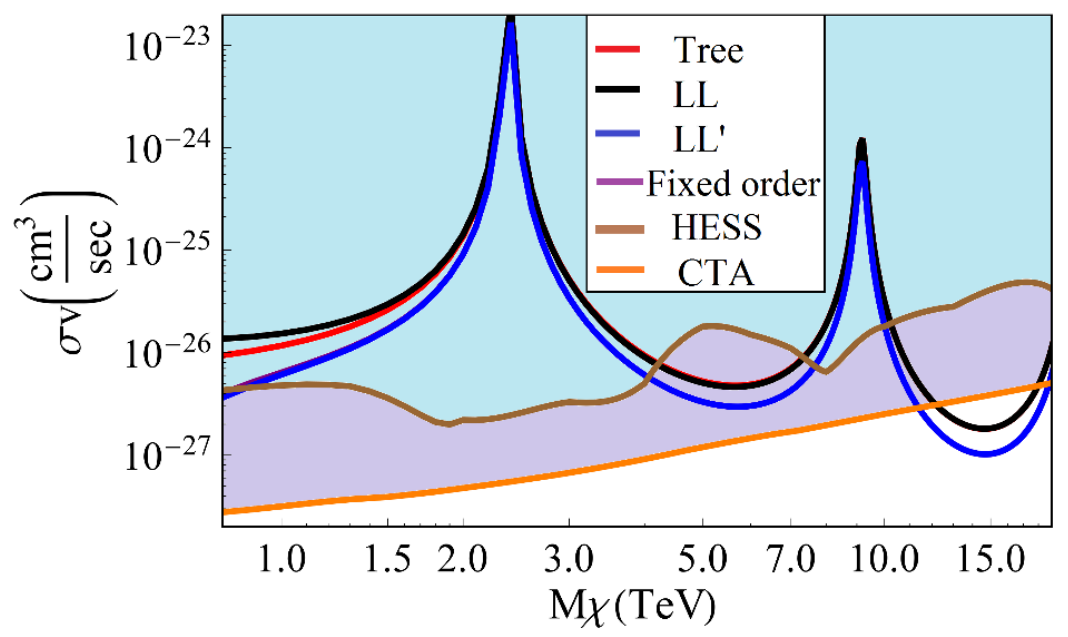

Figure 7. Wino annihilation cross section at $\mathrm{LL}^{\prime}$ with $z_{\mathrm{cut}}=0.85$. "Fixed order" takes out summation of the double logs, which is included in $\mathrm{LL}^{\prime}$, showing that this is a minor, $\mathcal{O}(1 \%)$, effect throughout this parameter range, and is thus barely visible on this logarithmic plot.

Figure 7 plots the wino cross section at LL'. ${ }^{9}$ This plot assumes a detector signal bin size of $0.15 M_{\chi}\left(z_{\text {cut }}=0.85\right)$. We see that the single-log terms produce a noticeable correction to the LL cross section. For example, at the thermal relic mass of $3 \mathrm{TeV}$, we have

$$
\begin{aligned}
\sigma_{\text {tree }} & =5.27 \times 10^{-26} \mathrm{~cm}^{3} / \mathrm{s} \\
\sigma_{\mathrm{LL}} & =5.07 \times 10^{-26} \mathrm{~cm}^{3} / \mathrm{s} \\
\sigma_{\mathrm{LL}^{\prime}} & =3.42 \times 10^{-26} \mathrm{~cm}^{3} / \mathrm{s},
\end{aligned}
$$

which indicates a $\sim 30 \%$ reduction.

A similar behavior is observed in the case of the higgsinos. Here, we give plots at two limiting cases in parameter space. The left panel of figure 8 shows the purest doublet that direct detection constraints allow. The neutral splitting, $\delta M_{n}=200 \mathrm{keV}$, with a chargino mass splitting $\delta M_{+}=350 \mathrm{MeV}$. For the MSSM, this would correspond to having gaugino masses $\sim 10^{8} \mathrm{GeV}$. The right panel uses $\delta M_{N}=2 \mathrm{GeV}$ and $\delta M_{+}=480 \mathrm{MeV}$, a spectrum we would get from gaugino mass parameters just a factor of a few larger. For $M_{\chi} \lesssim 3 \mathrm{TeV}$, we find again that the $\mathrm{LL}^{\prime}$ cross section is substantially reduced.

However, one thing to be noticed is that the full theory cross section contains single and double $\log \left(1-z_{\text {cut }}\right)$, which become large in the endpoint region. For our value of interest, $z_{\text {cut }}=0.85$, to get a handle on the importance of the endpoint logarithms, we see that $\log \left(1-z_{\text {cut }}\right)^{2}=\log \left(2 M_{\chi} / M_{W}\right)$ for $M_{\chi} \approx 1.4 \mathrm{TeV}$. In fact, we see in figure 8 that around this mass is where the $L^{\prime}$ curve stops tracking LL and turns to decrease sharply, going to negative values by $1 \mathrm{TeV}$. The corrections arising from these endpoint logarithms (which have only been partially captured in the present form of the EFT) can no longer

\footnotetext{
${ }^{9}$ The "fixed order" line expands the exponentials to first order and drops any other terms higher in $\alpha_{W}$. It thus provides a quantification of the effect of summing the double logs.
} 

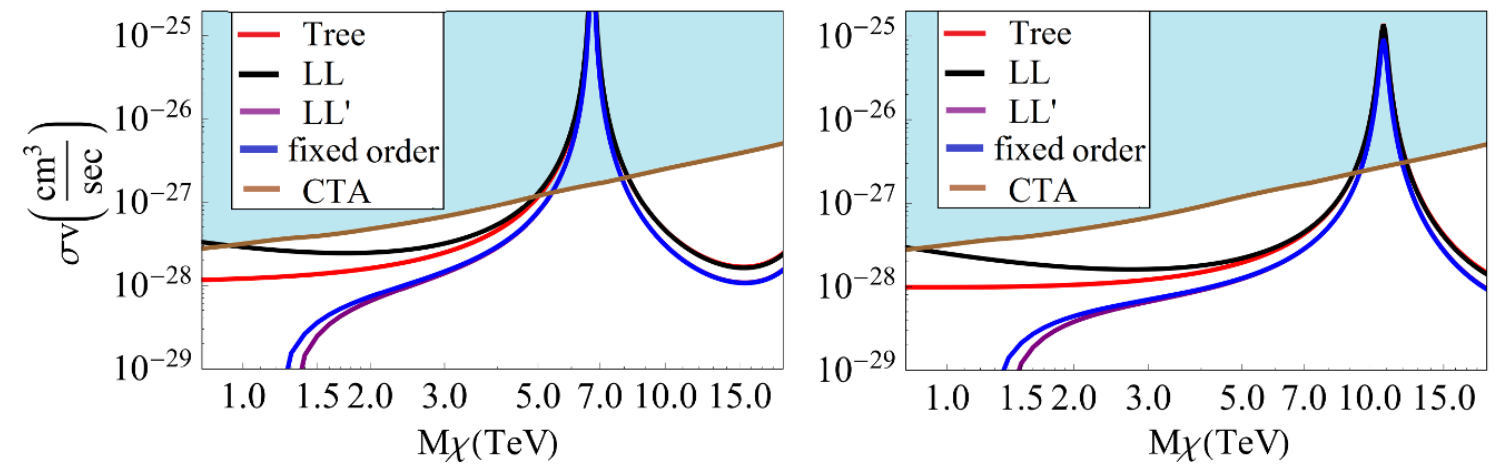

Figure 8. Higgsino annihilation cross section at $\mathrm{LL}^{\prime}$ with L: $\delta M_{N}=200 \mathrm{keV}, \delta M_{+}=350 \mathrm{MeV}$, R: $\delta M_{N}=2 \mathrm{GeV}, \delta M_{+}=480 \mathrm{MeV}$, and $z_{\text {cut }}=0.85$. Just as in figure 7, "fixed order" takes out summation of the double logs, which is included in $\mathrm{LL}^{\prime}$, showing that this is a minor effect throughout this parameter range.

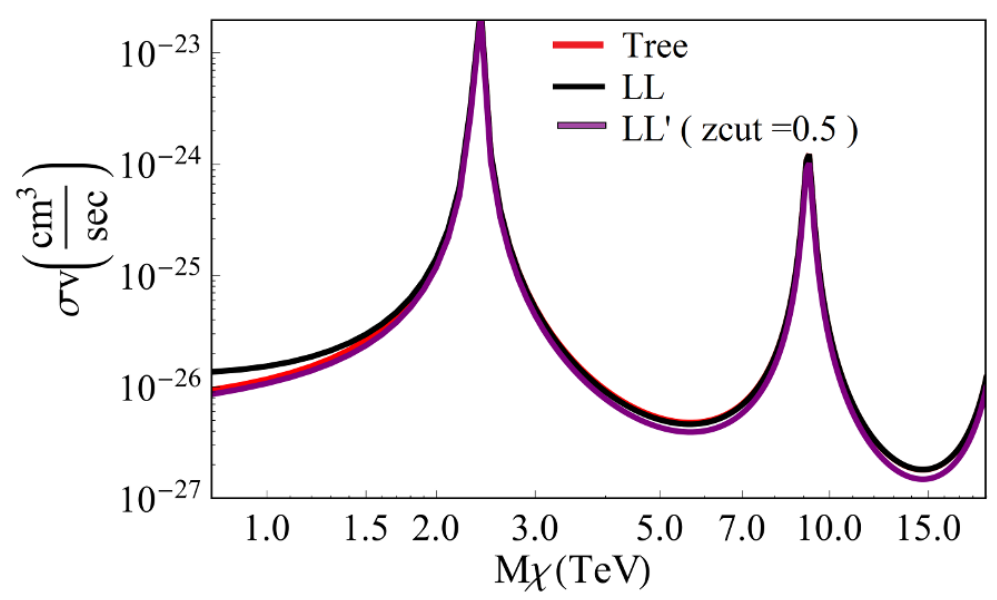

Figure 9. Wino annihilation cross section at LL' with a detector bin size of $0.5 M_{\chi}$.

be ignored. A full-theory tree-level calculation reveals that the missing terms are positive and enhance the cross section [25], as they must in order to restore positivity.

To test the hypothesis that the large corrections at $\mathrm{LL}^{\prime}$ arise from taking $z_{\text {cut }}$ close to the endpoint, we can take an unrealistically large "signal bin" with $z_{\text {cut }}=0.5$. In this calculation, endpoint logarithms are small and we are justified in dropping them completely. Additionally, with the larger bin, even though we smear out the photon energy at single-log order, a much greater fraction of them remain in our signal region. We see in figure 9 that LL' is indeed a small correction throughout our range. We get a very similar result with $z_{\text {cut }}=0.5$ for higgsinos, as well. This prompts us to conclude that as far as $\log \left(2 M_{\chi} / M_{W}\right)$ terms are concerned, we are justified in ignoring higher order corrections, such as the $z_{\text {cut }}$-independent two-loop cusp anomalous dimension, which would give a full NLL resummation. However, including effects that become large in the limit of small $1-z_{\text {cut }}$ will be important going forward. 
This exercise emphasized that it is vital to include endpoint corrections. Furthermore, as the resolution of future experiments improves, $z_{\text {cut }}$ will increase, and corrections will only get larger. Although our results here establish the need for further study, there are two conclusions we can draw already that are worth emphasizing:

1. Single logs won't save the wino: around the thermal relic mass of $3 \mathrm{TeV}, \log (1-$ $\left.z_{\text {cut }}\right)^{2} \lesssim \log \left(2 M_{\chi} / M_{W}\right)$, so endpoint corrections can be important. However, as we have mentioned they will be positive. Thus, our LL' result represents a floor for the wino annihilation rate, and we see that this is still an order of magnitude larger than the HESS exclusion limit. As we discussed [8], these limits are subject to large astrophysical uncertainties, and a sufficiently large, $>1 \mathrm{kpc}$, core can be invoked to reconcile the wino with experiment. However, we see that the situation is qualitatively unchanged from our earlier result, and saving the wino requires a profile in tension with current results from simulation [14-16].

2. The $1 \mathrm{TeV}$ thermal relic mass for the higgsino is at a tantalizing point: looking at the different curves at $1 \mathrm{TeV}$ in figure 8 , we see a significant change in the result at each order in the calculation. Furthermore, there is an additional large change still to be determined as endpoint corrections will be needed to restore a positive cross section. We see that Sommerfeld enhancement and including large Sudakov and endpoint logarithms will all be necessary to give a controlled result. The fact that the LL curve touches the projected CTA (the successor experiment to HESS) limits at $1 \mathrm{TeV}$ only deepens the intrigue. The possibility for the next generation of experiments to probe this motivated limit hangs in the balance.

To proceed with the analysis requires a two-step EFT. This involves matching the full theory to a SCET-I theory with a power counting parameter $\eta=1-z_{\text {cut }}$, followed by matching to the SCET-II EFT developed in this paper with the modified parameter $\lambda^{\prime}=M_{W} /\left[M_{\chi}\left(1-z_{\text {cut }}\right)\right]$. In the SCET-I theory, we can no longer integrate out the recoil state into the coefficient of a local operator, as described in the introduction to section 2. This is because we need to track the separation between $\left(1-z_{\text {cut }}\right) M_{\chi}$ and $M_{\chi}$, a hierarchy that is missed by lumping all scales $\gg \lambda^{2} M_{\chi}^{2}$ together. We will analyze this topic in a forthcoming paper.

\section{Acknowledgments}

We thank Grigory Ovanesyan, Matthew Reece, Ira Rothstein, Tracy Slatyer, Iain Stewart and Brock Tweedie for discussions. The authors are supported by DOE grants DOE DE-FG02-04ER41338 and FG-02-06ER41449. MB is also supported by DOE grant DESC0003883.

\section{A Neutralino masses and mixing}

For more details on the neutralino spectrum in the limit of nearly-pure higgsino LSP, we refer the reader to the discussion in [24]. In the gauge-eigenstate basis $\chi^{0}=\left(\tilde{B}, \tilde{W}^{0}, \tilde{h}_{d}^{0}, \tilde{h}_{u}^{0}\right)$, 
the neutralino mass part of the Lagrangian is

$$
\mathcal{L} \supset-\frac{1}{2}\left(\chi^{0}\right)^{T} M_{\tilde{N}} \chi^{0}+\text { c.c. },
$$

where

$$
M_{\tilde{N}}=\left(\begin{array}{cccc}
M_{1} & 0 & -c_{\beta} s_{W} m_{Z} & s_{\beta} s_{W} m_{Z} \\
0 & M_{2} & c_{\beta} c_{W} m_{Z} & -s_{\beta} c_{W} m_{Z} \\
-c_{\beta} s_{W} m_{Z} & c_{\beta} c_{W} m_{Z} & 0 & -\mu \\
s_{\beta} s_{W} m_{Z} & -s_{\beta} c_{W} m_{Z} & -\mu & 0
\end{array}\right)
$$

where $s_{\beta}=\sin \beta, c_{W}=\cos \theta_{W}$, etc. Following [32], we expand the eigenvalues in the limit that $m_{Z} \ll \mu, M_{1}, M_{2}$. For the case of higgsino LSP, we can additionally expand in $\mu / M_{1,2}$, as we will always consider scenarios with at least a factor of a few hierarchy to avoid large mixing effects. In this case, the neutralino mass eigenststes are very nearly a bino-like $\tilde{N}_{1} \approx \tilde{B}$; a wino-like $\tilde{N}_{2} \approx \tilde{W}^{0}$; and higgsino-like $\tilde{N}_{3}, \tilde{N}_{4} \approx\left(\tilde{h}_{u}^{0} \pm \tilde{h}_{d}^{0}\right) / \sqrt{2}$, with mass eigenvalues:

$$
\begin{aligned}
m_{\tilde{N}_{1}} & \approx M_{1}-\frac{m_{z}^{2} s_{W}^{2}\left(M_{1}+\mu \sin 2 \beta\right)}{\mu^{2}-M_{1}^{2}} \approx M_{1}+\frac{m_{z}^{2} s_{W}^{2}}{M_{1}} \\
m_{\tilde{N}_{2}} & \approx M_{2}-\frac{m_{W}^{2}\left(M_{2}+\mu \sin 2 \beta\right)}{\mu^{2}-M_{2}^{2}} \approx M_{2}+\frac{m_{W}^{2}}{M_{2}} \\
m_{\tilde{N}_{3}}, m_{\tilde{N}_{4}} & =|\mu|+\frac{m_{z}^{2}(I-\sin 2 \beta)\left(\mu+M_{1} c_{W}^{2}+M_{2} s_{W}^{2}\right)}{2\left(\mu+M_{1}\right)\left(\mu+M_{2}\right)} \\
& \approx|\mu|+\frac{m_{z}^{2}(I-\sin 2 \beta)\left(M_{1} c_{W}^{2}+M_{2} s_{W}^{2}\right)}{2 M_{1} M_{2}}, \\
& =|\mu|+\frac{m_{z}^{2}(I+\sin 2 \beta)\left(\mu-M_{1} c_{W}^{2}-M_{2} s_{W}^{2}\right)}{2\left(\mu-M_{1}\right)\left(\mu-M_{2}\right)} \\
& \approx|\mu|-\frac{m_{z}^{2}(I+\sin 2 \beta)\left(M_{1} c_{W}^{2}+M_{2} s_{W}^{2}\right)}{2 M_{1} M_{2}}
\end{aligned}
$$

where $M_{1}$ and $M_{2}$ are real and positive and $\mu$ is real with $I= \pm 1$. A similar analysis for the chargino reveals mass eigenvalues for the wino-like and higgsino-like chargino

$$
\begin{aligned}
& m_{\tilde{C}_{1}}=M_{2}-\frac{m_{W}^{2}\left(M_{2}+\mu \sin 2 \beta\right)}{\mu^{2}-M_{2}^{2}} \approx M_{2}+\frac{m_{W}^{2}}{M_{2}} \\
& m_{\tilde{C}_{2}}=|\mu|+\frac{m_{W}^{2} I\left(\mu+M_{2} \sin 2 \beta\right)}{\mu^{2}-M_{2}^{2}} \approx|\mu|+\frac{m_{W}^{2} \sin 2 \beta}{M_{2}}
\end{aligned}
$$

For the case $I=-1$, and $M_{1} \sim M_{2}$ we get a mass splitting between the higgsino-like neutral states to be

$$
\Delta M=\frac{m_{Z}^{2}}{M_{1}}
$$

This mass difference is of the order of $10-100 \mathrm{KeV}$ for $M_{2} \sim 10^{9}-10^{8} \mathrm{GeV}$. For direct detection experiments, the LSP can scatter into the heavier neutralino via a $Z$ exchange with 
a nucleus in the detector. Current experiments such as XENON10 [28], XENON100 [29] and LUX [30] have sensitivities to $\Delta M_{N} \leq(120-200) \mathrm{keV}$ in the case of higgsino DM. This sets the lower limit for the mass splitting and requires $M_{1}, M_{2} \leq 10^{5} \mathrm{TeV}$.

The LSP $\left(\tilde{N}_{3}\right)$ and the higgsino-like chargino $\left(\tilde{C}_{2}\right)$ are almost degenerate with a mass splitting

$$
\Delta M_{+}=\frac{2 M_{W}^{2} \sin 2 \beta+M_{z}^{2}(1+\sin 2 \beta)}{2 M_{1}},
$$

which is again in the range of $100 \mathrm{~s}$ of $\mathrm{keV}$ for $M_{1}=M_{2} \approx 10^{5} \mathrm{TeV}$. However, these are only the tree level values. It turns out that after including radiative corrections, $\Delta M_{N}$ remains of the same order, while $\Delta M_{+}$is modified substantially [31]. This pushes up the mass splitting to $\sim 350 \mathrm{MeV}$ asymptotically in the large gaugino mass limit.

\section{B Sommerfeld enhancement}

\section{B.1 Wino}

In order to quantify the semi-inclusive rate calculation, we need to determine the wavefunctions-at-the-origin (Sommerfeld enhancement factors) that enter our final, LL' cross sections in eqs. (2.19) and (3.24). They can be computed in principle in the nonrelativistic effective theory by summing the ladder exchange of electroweak gauge bosons between neutralinos to all orders. Fortunately, this is equivalent to the operationally simpler task of solving the Schrödinger equation for our two, two-body states $\left|\chi^{0} \chi^{0}\right\rangle$ and $\left|\chi^{+} \chi^{-}\right\rangle$in the presence of the electroweak potential [23, 26, 27]. Since it contains Coulomb, Yukawa, and mass-shift pieces and is off-diagonal for the two states, we solve it numerically, in a manner similar to [5]. As expected for slowly moving particles in the presence of an attractive potential, we find Sommerfeld enhancement for the annihilation. For some regions of $M_{\chi}$, this is orders of magnitude above the perturbative rate.

Taking into account appropriate state normalization, the Schrödinger potential is

$$
V(r)=\left(\begin{array}{cc}
2 \delta M-\frac{\alpha}{r}-\alpha_{W} c_{W}^{2} \frac{e^{-m_{Z^{r}}}}{r} & -\sqrt{2} \alpha_{W} \frac{e^{-m_{W^{r}}}}{r} \\
-\sqrt{2} \alpha_{W} \frac{e^{-m_{W}}}{r} & 0
\end{array}\right),
$$

where $\delta M \equiv M_{\chi^{+}}-M_{\chi^{0}}$. For numerical analysis, we use $\delta M=0.17 \mathrm{GeV}$, which is its value over much of MSSM parameter space. We refer the reader to [8] for details about these enhancement factors for the wino.

\section{B.2 Higgsino}

For the case of the higgsino, we have three possible channels of annihilation to photons, the LSP neutralino, the heavier neutralino, and the chargino. The initial LSP state can oscillate into the heavier neutralino via $Z$ exchange or to the chargino state via the $W$ boson. Taking into account appropriate state normalization, the Schrödinger potential is

$$
V(r)=\left(\begin{array}{ccc}
2 \delta m-\frac{\alpha}{r}-\frac{\alpha_{W}\left(1-2 c_{W}^{2}\right)^{2}}{4 c_{W}^{2}} \frac{e^{-m_{Z^{r}}}}{r} & -\sqrt{2} \alpha_{W} \frac{e^{-m_{W}}}{r} & -\sqrt{2} \alpha_{W} \frac{e^{-m_{W} r}}{4 r} \\
-\sqrt{2} \alpha_{W} \frac{e^{-m_{W}}}{4 r} & 0 & -\frac{\alpha_{W} e^{-m_{Z^{r}}}}{4 c_{W}^{2} r} \\
-\sqrt{2} \alpha_{W} \frac{e^{-m_{W}}}{4 r} & -\frac{\alpha_{W} e^{-m_{Z^{r}}}}{4 c_{W}^{2}} & 2 \delta m_{N}
\end{array}\right),
$$



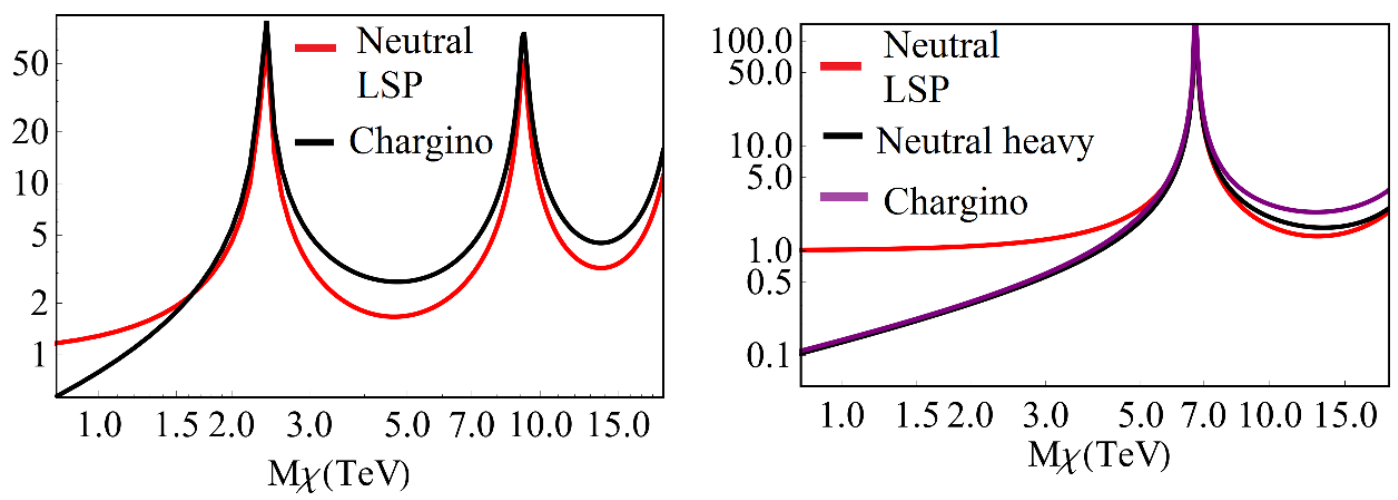

Figure 10. Sommerfeld enhancement factors for wino (higgsino) in the left (right) panel. For the higgsino case, we have $\Delta M=200 \mathrm{keV}$ and $\Delta M_{+}=350 \mathrm{MeV}$.

We plot our numerically determined Sommerfeld factors in figure 10 . These are the $\psi_{1-3}(0)$ in eqs. (2.21) and (3.23). Interestingly, we find that at the thermal relic mass, $1 \mathrm{TeV}$, the charged-state wavefunction-squared is $\sim 10^{-2}$. Since charged annihilation is tree-level, we find that it is comparable to the neutral-state annihilation that occurs at one loop. We see this reflected in the higgsino annihilation plotted in figure 8.

\section{Photon wavefunction renormalization}

The wavefunction renormalization of the photon is the residue of the pole of the two-point function obtained after applying radiative corrections to $\Pi_{\gamma \gamma}$ (figure 6). In the preceding calculation, we have put a generic IR cut-off at the scale $M_{W}$ which then gives us a correction of the form $\beta_{0} \log \left(\frac{2 M_{\chi}}{M_{W}}\right)$. While this will work for the gauge bosons and scalars which have masses around the electroweak scale, it will clearly not do for the fermionic loops. This is due to the fact that since the photon is massless, the IR cutoff for the loop integrals is the mass of the particle in the loop. Thus, the contribution of the fermions needs to be modified to give $\sim \log \left(\frac{2 M_{\chi}}{m_{f}}\right)$. While this is fine for the case of leptons and heavy quarks, the case of the light quarks is not so clear due to the non-perturbative physics involved.

In principle, the way to do it formally is to match the EFT in the present form to an EFT below the electroweak scale. Such an EFT will necessarily have the SU(2) symmetry broken, with the $W$ and $Z$ bosons integrated out. The degrees of freedom that remain are the light fermions and the photon (along with the initial state winos/higgsinos). Since we are only computing diagrams up to one loop, the photon fragmentation function in this EFT will receive only a virtual correction which is the photon self energy correction $\Pi_{\gamma \gamma}$ involving light fermionic loops. This in turn tells us that operationally the way to implement this correction in the final cross section is to identify the photon self energy terms and add $\Pi_{\gamma \gamma}$ evaluated at the scale $M_{W}$. Such terms are easy enough to identify since they contribute to the beta function of both the $\mathrm{SU}(2)$ and hypercharge gauge coupling. Thus we modify 
our expression for the beta function as

$$
\frac{\alpha^{i}}{2 \pi} \beta_{0}^{i} \log \left(\frac{2 M_{\chi}}{M_{W}}\right) \rightarrow \frac{\alpha^{i}}{2 \pi} \beta_{0}^{i} \log \left(\frac{2 M_{\chi}}{M_{W}}\right)+\Pi_{\gamma \gamma}\left(M_{W}^{2}\right)
$$

where $\alpha^{i}$ can be either $\alpha_{W}$ or $\alpha^{\prime}$ with the corresponding $\beta$ functions, $\beta_{0}$ or $\beta_{0}^{\prime}$. We use this to modify our final $\mathrm{LL}^{\prime}$ rate expressions, eqs. (2.19) and (3.24). We get such a simple form in eq. (C.1) because our operators in the unbroken theory already explicitly project onto a photon external state. Thus, they already contain the appropriate $\sin \theta_{W}$ factors needed for going to the broken theory below $M_{W}$ and no further rotation is needed.

Since $\Pi_{\gamma \gamma}$ involves non perturbative QCD corrections, we cannot compute it analytically. There is however, a method of obtaining the contribution of the quarks to the photon self-energy [33]: the evaluation of the loop diagrams can be related to the crosssection measurements. The imaginary part of $\Pi_{\gamma \gamma}$ for hadrons is directly related to $R_{\text {had }}$, the QED cross-section of the process $e^{+} e^{-} \rightarrow$ hadrons normalized to the QED cross section for muon pair production.

$$
\operatorname{Im} \Pi_{\gamma \gamma}(s)=-\frac{\alpha}{3} R_{h a d}(s)
$$

The real part of $\Pi_{\gamma \gamma}$ is obtained by using the Kramers-Kronig rule. Since we have included a $\log \left(\frac{2 M_{\chi}}{M_{W}}\right)$ factor, the missing pieces can be recovered by adding the leptonic and hadronic contributions below the scale $M_{W}$. For the $\Pi_{\gamma \gamma}$ in eqs. (2.19) and (3.24), we therefore simply get $\Pi_{\gamma \gamma}=\alpha(0) / \alpha\left(M_{Z}\right)-1$, where $\alpha$ is the fine-structure constant. This contribution has been evaluated numerically to be -0.0594 .

Open Access. This article is distributed under the terms of the Creative Commons Attribution License (CC-BY 4.0), which permits any use, distribution and reproduction in any medium, provided the original author(s) and source are credited.

\section{References}

[1] S. Dimopoulos, LHC, SSC and the universe, Phys. Lett. B 246 (1990) 347 [InSPIRE].

[2] J. Fan and M. Reece, In Wino Veritas? Indirect Searches Shed Light on Neutralino Dark Matter, JHEP 10 (2013) 124 [arXiv:1307.4400] [INSPIRE].

[3] J. Hisano, S. Matsumoto, M. Nagai, O. Saito and M. Senami, Non-perturbative effect on thermal relic abundance of dark matter, Phys. Lett. B 646 (2007) 34 [hep-ph/0610249] [INSPIRE].

[4] M. Cirelli, A. Strumia and M. Tamburini, Cosmology and Astrophysics of Minimal Dark Matter, Nucl. Phys. B 787 (2007) 152 [arXiv:0706.4071] [INSPIRE].

[5] T. Cohen, M. Lisanti, A. Pierce and T.R. Slatyer, Wino Dark Matter Under Siege, JCAP 10 (2013) 061 [arXiv:1307.4082] [inSPIRE].

[6] HESS collaboration, A. Abramowski et al., Search for Photon-Linelike Signatures from Dark Matter Annihilations with H.E.S.S., Phys. Rev. Lett. 110 (2013) 041301 [arXiv:1301.1173] [INSPIRE]. 
[7] M. Baumgart, I.Z. Rothstein and V. Vaidya, Calculating the Annihilation Rate of Weakly Interacting Massive Particles, Phys. Rev. Lett. 114 (2015) 211301 [arXiv:1409.4415] [INSPIRE].

[8] M. Baumgart, I.Z. Rothstein and V. Vaidya, Constraints on Galactic Wino Densities from Gamma Ray Lines, JHEP 04 (2015) 106 [arXiv:1412.8698] [INSPIRE].

[9] W.E. Caswell and G.P. Lepage, Effective Lagrangians for Bound State Problems in QED, QCD and Other Field Theories, Phys. Lett. B 167 (1986) 437 [INSPIRE].

[10] C.W. Bauer, S. Fleming and M.E. Luke, Summing Sudakov logarithms in $B \rightarrow X_{s} \gamma$ in effective field theory, Phys. Rev. D 63 (2000) 014006 [hep-ph/0005275] [INSPIRE].

[11] C.W. Bauer, S. Fleming, D. Pirjol and I.W. Stewart, An Effective field theory for collinear and soft gluons: Heavy to light decays, Phys. Rev. D 63 (2001) 114020 [hep-ph/0011336] [INSPIRE].

[12] C.W. Bauer, D. Pirjol and I.W. Stewart, Soft collinear factorization in effective field theory, Phys. Rev. D 65 (2002) 054022 [hep-ph/0109045] [INSPIRE].

[13] C.W. Bauer, S. Fleming, D. Pirjol, I.Z. Rothstein and I.W. Stewart, Hard scattering factorization from effective field theory, Phys. Rev. D 66 (2002) 014017 [hep-ph/0202088] [INSPIRE].

[14] A. Di Cintio et al., The dependence of dark matter profiles on the stellar-to-halo mass ratio: a prediction for cusps versus cores, Mon. Not. Roy. Astron. Soc. 437 (2014) 415 [arXiv: 1306.0898] [INSPIRE].

[15] M. Kuhlen, J. Guedes, A. Pillepich, P. Madau and L. Mayer, An Off-center Density Peak in the Milky Way's Dark Matter Halo?, Astrophys. J. 765 (2013) 10 [arXiv:1208.4844] [INSPIRE].

[16] F. Marinacci, R. Pakmor and V. Springel, The formation of disc galaxies in high resolution moving-mesh cosmological simulations, Mon. Not. Roy. Astron. Soc. 437 (2014) 1750 [arXiv: 1305.5360] [INSPIRE].

[17] M. Bauer, T. Cohen, R.J. Hill and M.P. Solon, Soft Collinear Effective Theory for Heavy WIMP Annihilation, JHEP 01 (2015) 099 [arXiv: 1409.7392] [INSPIRE].

[18] G. Ovanesyan, T.R. Slatyer and I.W. Stewart, Heavy Dark Matter Annihilation from Effective Field Theory, Phys. Rev. Lett. 114 (2015) 211302 [arXiv:1409.8294] [INSPIRE].

[19] I. Rothstein, Effective Field Theory, to appear.

[20] M. Baumgart and V. Vaidya, Constraints on Galactic Wino Densities from Gamma Ray Lines, to appear.

[21] J.-Y. Chiu, A. Jain, D. Neill and I.Z. Rothstein, A Formalism for the Systematic Treatment of Rapidity Logarithms in Quantum Field Theory, JHEP 05 (2012) 084 [arXiv:1202.0814] [INSPIRE].

[22] J.-y. Chiu, A. Jain, D. Neill and I.Z. Rothstein, The Rapidity Renormalization Group, Phys. Rev. Lett. 108 (2012) 151601 [arXiv:1104.0881] [INSPIRE].

[23] J. Hisano, S. Matsumoto, M.M. Nojiri and O. Saito, Non-perturbative effect on dark matter annihilation and gamma ray signature from galactic center, Phys. Rev. D 71 (2005) 063528 [hep-ph/0412403] [INSPIRE]. 
[24] N. Nagata and S. Shirai, Higgsino Dark Matter in High-Scale Supersymmetry, JHEP 01 (2015) 029 [arXiv: 1410.4549] [INSPIRE].

[25] L. Bergstrom, T. Bringmann, M. Eriksson and M. Gustafsson, Gamma rays from heavy neutralino dark matter, Phys. Rev. Lett. 95 (2005) 241301 [hep-ph/0507229] [INSPIRE].

[26] R. Iengo, Sommerfeld enhancement: General results from field theory diagrams, JHEP 05 (2009) 024 [arXiv: 0902.0688] [INSPIRE].

[27] S. Cassel, Sommerfeld factor for arbitrary partial wave processes, J. Phys. G 37 (2010) 105009 [arXiv: 0903.5307] [INSPIRE].

[28] XENON10 collaboration, J. Angle et al., Constraints on inelastic dark matter from XENON10, Phys. Rev. D 80 (2009) 115005 [arXiv:0910.3698] [INSPIRE].

[29] XENON100 collaboration, E. Aprile et al., Dark Matter Results from 225 Live Days of XENON100 Data, Phys. Rev. Lett. 109 (2012) 181301 [arXiv:1207.5988] [InSPIRE].

[30] LUX collaboration, D.S. Akerib et al., First results from the LUX dark matter experiment at the Sanford Underground Research Facility, Phys. Rev. Lett. 112 (2014) 091303 [arXiv: 1310.8214] [INSPIRE].

[31] D.M. Pierce, J.A. Bagger, K.T. Matchev and R.-j. Zhang, Precision corrections in the minimal supersymmetric standard model, Nucl. Phys. B 491 (1997) 3 [hep-ph/9606211] [INSPIRE].

[32] S.P. Martin, A supersymmetry primer, hep-ph/9709356 [INSPIRE].

[33] H. Burkhardt and B. Pietrzyk, Update of the hadronic contribution to the QED vacuum polarization, Phys. Lett. B 513 (2001) 46 [INSPIRE]. 